\title{
Autistic-like phenotypes in Cadps2-knockout mice and aberrant CADPS2 splicing in autistic patients
}

\author{
Tetsushi Sadakata, ${ }^{1}$ Miwa Washida, ${ }^{1}$ Yoshimi Iwayama, ${ }^{2}$ Satoshi Shoji, ${ }^{1}$ Yumi Sato, ${ }^{1}$ \\ Takeshi Ohkura, ${ }^{3}$ Ritsuko Katoh-Semba, ${ }^{4}$ Mizuho Nakajima, ${ }^{2}$ Yukiko Sekine, ${ }^{1}$ Mika Tanaka, ${ }^{5}$ \\ Kazuhiko Nakamura, ${ }^{6}$ Yasuhide Iwata, ${ }^{6}$ Kenji J. Tsuchiya, ${ }^{6}$ Norio Mori, ${ }^{6}$ Sevilla D. Detera-Wadleigh,7 \\ Hironobu Ichikawa, ${ }^{3}$ Shigeyoshi Itohara, ${ }^{8}$ Takeo Yoshikawa, $^{2}$ and Teiichi Furuichi ${ }^{1}$ \\ 'Laboratory for Molecular Neurogenesis and 2Laboratory for Molecular Psychiatry, RIKEN Brain Science Institute, Saitama, Japan. \\ ${ }^{3}$ Tokyo Metropolitan Umegaoka Hospital, Tokyo, Japan. ${ }^{4}$ Department of Perinatology, Institute for Developmental Research, \\ Aichi Human Service Center, Kasugai, Japan. ${ }^{5}$ Research Resource Center, RIKEN Brain Science Institute, Saitama, Japan. \\ ${ }^{6}$ Department of Psychiatry and Neurology, Hamamatsu University School of Medicine, Hamamatsu, Japan. ${ }^{7}$ Mood and Anxiety Disorders Program, \\ National Institute of Mental Health, Bethesda, Maryland, USA. ${ }^{8}$ Laboratory for Behavioral Genetics, RIKEN Brain Science Institute, Saitama, Japan.
}

\begin{abstract}
Autism, characterized by profound impairment in social interactions and communicative skills, is the most common neurodevelopmental disorder, and its underlying molecular mechanisms remain unknown. $\mathrm{Ca}^{2+}$ dependent activator protein for secretion 2 (CADPS2; also known as CAPS2) mediates the exocytosis of densecore vesicles, and the human CADPS2 is located within the autism susceptibility locus 1 on chromosome $7 \mathrm{q}$. Here we show that Cadps2-knockout mice not only have impaired brain-derived neurotrophic factor release but also show autistic-like cellular and behavioral phenotypes. Moreover, we found an aberrant alternatively spliced CADPS2 mRNA that lacks exon 3 in some autistic patients. Exon 3 was shown to encode the dynactin 1binding domain and affect axonal CADPS2 protein distribution. Our results suggest that a disturbance in CADPS2-mediated neurotrophin release contributes to autism susceptibility.
\end{abstract}

\section{Introduction}

Autism is a severe neurodevelopmental disorder marked by profound disturbances in social, communicative, and behavioral functioning $(1,2)$. Epidemiological studies have shown that the prevalence of autism spectrum disorders is 3-6 per 1,000, with a male-to-female ratio of $3: 1$ (3). The concordance rate is about $90 \%$ in monozygotic twins and $10 \%$ in dizygotic twins (4), suggesting that autism has a prominent genetic component. Several genes, such as neuroligin 3 (NLGN3), NLGN4, and PTEN, have been suggested to be associated with the development of autism $(3,5,6)$. One susceptibility locus for autism was mapped to human chromosome 7q31-q33 (autism susceptibility locus 1 [AUTS1]) (7); however, none of the several candidate genes mapped to the AUTS1 locus have been directly implicated in autism (3).

$\mathrm{Ca}^{2+}$-dependent activator protein for secretion 2 (CADPS2) is a member of the CAPS/CADPS protein family that regulates the exocytosis of dense-core vesicles at the ATP-dependent priming step by binding both phosphatidyl inositol 4,5-bisphosphate and dense-core vesicles (8). The human CADPS2 is located on chromosome 7q31.32, within the AUTS1 locus (9). Mouse CADPS2 protein is associated with brain-derived neurotrophic factor-containing (BDNF-containing) secretory vesicles and is involved in the activity-dependent release of BDNF (10), and the cellular distribution of CADPS 2 in the mouse brain largely coincides with that of BDNF (11). BDNF plays a key role in many aspects of brain

Nonstandard abbreviations used: BDNF, brain-derived neurotrophic factor; CADPS2, $\mathrm{Ca}^{2+}$-dependent activator protein for secretion 2; DIV, days in vitro; LD, light/dark.

Conflict of interest: The authors have declared that no conflict of interest exists. Citation for this article: J. Clin. Invest. 117:931-943 (2007). doi:10.1172/JCI29031. development and function, including the formation of synapses and circuits $(12,13)$. However, the detailed mechanism of BDNF secretion remains elusive (14). A recent study indicates that the decreased level of BDNF expression in methyl CpG-binding protein 2-mutant (Mecp2-mutant) mice, a model of Rett syndrome (15), affects disease progression (16).

In this report, we describe the association of a deficit in CADPS2-mediated neurotrophin release with autism susceptibility by analyzing Cadps2-knockout mice and detecting aberrant alternative splicing in CADPS2 mRNA in autistic patients. Our results suggest that defects of CADPS2 function might contribute to autism susceptibility.

\section{Results}

The mouse Cadps 2 gene was disrupted by deleting $0.9 \mathrm{~kb}$ of the first exon from its genomic sequence (Figure 1A), which was confirmed by Southern blot hybridization (for genomic structure, see Figure 1B) and RT-PCR (for mRNA expression, see Figure 1C). WT $\left(\right.$ Cadps $\left.2^{+/+}\right)$, heterozygous (Cadps $2^{+/-}$), and homozygous (Cadps $2^{-/-}$) pups were born at the expected 1:2:1 Mendelian frequency $\left(66^{+/+}, 128^{+/-}\right.$, and $63^{-/-}$of 257 animals analyzed). No CADPS 2 protein was detected in the cerebellum, neocortex, or hippocampus of Cadps $2^{-/-}$mice, but a reduced amount was present in those of Cadps $2^{+/-}$mice in relation to WT mice (Figure 1D). Cadps2 $2^{-/-}$mice in standard breeding cages exhibited no obvious difference in life expectancy from control mice, and both male and female Cadps $2^{-/-}$mice had normal reproductive ability. No significant change in appearance of Cadps $2^{-/-}$mice was observed except for decreased body weight (Supplemental Figure 1; supplemental material available online with this article; doi:10.1172/JCI29031DS1). 


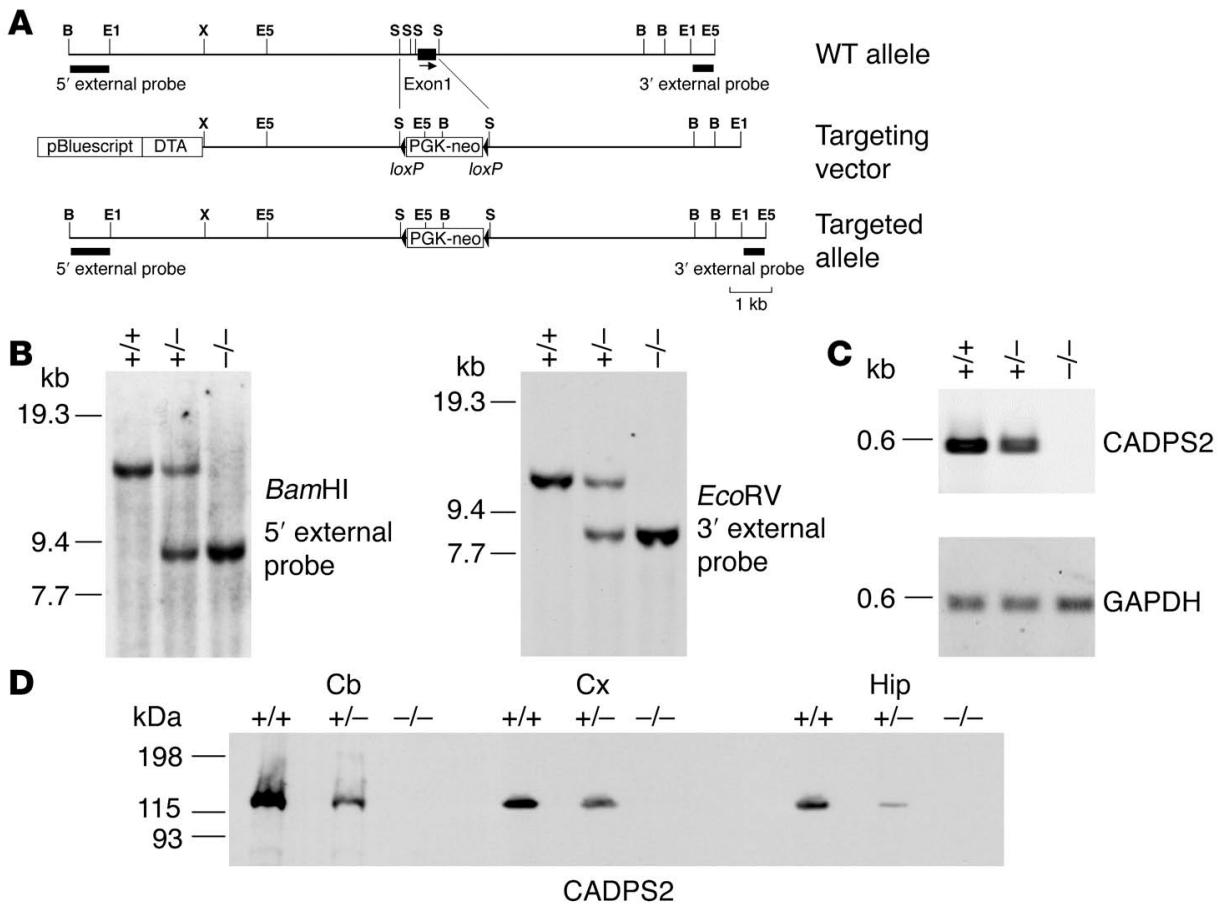

Figure 1

Generation of Cadps2 $2^{-/}$mice. (A) Maps of the Cadps2 gene, the targeting vector, and the resultant targeted allele are shown. Probes for Southern blot analysis to screen for targeted ES clones are indicated by small solid bars. Restriction enzyme sites include the following: B, BamHI; E1, EcoRl; E5, EcoRV; S, Smal; X, Xhol. DTA, diphtheria toxin A fragment (B) Southern blot analysis, using the probes indicated in A, of DNA from tails of a single litter derived from a cross between heterozygotes. +/+, WT mice; +/-, heterozygous mice; -/-, homozygous mice. (C) RT-PCR banding patterns of CADPS2 using brain total RNA of P21 WT, heterozygous, and homozygous mice. The GAPDH gene was used as a constantly expressed control mRNA. (D) Immunoblot analysis of the cerebellum, neocortex, and hippocampus of P8 WT, heterozygous, and homozygous mice. Protein lysates from these brain regions were immunoblotted with anti-CADPS2 antibody. Cb, cerebellum; Cx, neocortex; Hip, hippocampus.

We first analyzed the effect of loss of CADPS2 on basal sensory and cognitive performances of Cadps2 $2^{-/}$mice before detailed behavioral examination. Cadps $2^{-/-}$mice showed no significant difference in a visual test (visual recognition of surface to hold under tail suspension), olfactory test (scenting out hidden food), and auditory test (movements to sound stimulus) in comparison with their WT littermates (Supplemental Table 1). The Morris water maze test was performed to evaluate cognitive function of Cadps $2^{--}$mice (Supplemental Figure 2). Escape latency to climb onto a visible platform in a pool was almost the same between WT and Cadps2 $2^{-/}$mice (Supplemental Figure 2A), indicating that there are no significant differences in their motor function and motivation required for this task. After 4 days of training mice to find a hidden platform, latency to reach the platform was slightly improved in Cadps $2^{--}$mice but did not differ significantly from that in WT mice $(P=0.106$; 2 -way ANOVA with repeated measures) (Supplemental Figure 2B). By removing the platform after the last hidden platform trial, memory of the previous platform location was analyzed the next day (probe test). Interestingly, Cadps $2^{--}$mice showed lower spatial accuracy in exploring for the platform location than WT mice $(P<0.05$; Student's $t$ test), indicating impairment in retention of spatial memory in Cadps $2^{-/-}$mice (Supplemental Figure 2C).
Autism is characterized by abnormal behavioral characteristics, including impaired social interaction $(1,2)$, hyperactivity $(17,18)$, and augmented anxiety and/or reduced environmental exploration in a novel environment $(1,2,19)$, and is frequently accompanied by an abnormal sleep-wake rhythm $(20,21)$. Detailed behavioral analyses demonstrated several autistic-like behavioral phenotypes in Cadps $2^{-1-}$ mice. We used male mice for the analyses of behavior but used female mice for the analysis of maternal behavior.

Firstly, the social interaction of Cadps $2^{-/-}$mice was impaired. Pairs of mice of the same genotype (Cadps $2^{+/+}$ or Cadps $\left.2^{--}\right)$that had never met were placed in a neutral cage for $20 \mathrm{~min}$ utes, and the frequency of interactions by Cadps $2^{-/-}$mice was significantly lower than that of WT mice $(P<0.05$; Student's $t$ test; Figure 2A). Secondly, the Cadps2-广- mice exhibited hyperactivity in their home cages. When locomotor activity was measured over a 6-day period (12-hour light/12-hour dark cycle [12-hour LD cycle]) after habituation to a fresh cage for 24 hours, the home-cage activity of the Cadps $2^{-/-}$ mice was significantly greater than that of their WT littermates $(P<0.05$; Student's $t$ test; Figure 2B). This difference between WT and Cadps2-/mice was observed only in the dark cycle, during which mice are more active in general (Supplemental Figure 3).

Thirdly, the Cadps2 $2^{-/-}$mice tended to show decreased exploratory behavior and/or increased anxiety in a novel environment. Cadps $2^{-1-}$ mice placed in an open field in the light cycle showed normal locomotor activity in comparison with their WT littermates, during each 5-minute block (Figure 2C). There was no significant difference between WT $(13.6 \pm 2.0 \%$, mean $\pm \mathrm{SEM} ; n=17)$ and Cadps $2^{-/-}$mice $(10.7 \pm 2.0 \%$, mean $\pm \mathrm{SEM} ; n=15)$ in the time spent in a central area, which is generally accepted as a measure of fear in rodents (22). Moreover, Cadps $2^{-/-}$mice showed no significant anxiety-like behavior in the LD transition test (Supplemental Figure 4, A and B). However, as shown in Figure 2D, Cadps2-/- mice became less active than WT mice during 3 time blocks (a total of 15 minutes) during which they were placed in an open field containing a novel object (the black and white vertical object shown in the inset of Figure 2D) at its center $(P<0.01$; Student's $t$ test). Representative traces from WT and Cadps2 $2^{-/-}$mice are shown in Figures 2, E and F, respectively. The Cadps2 $2^{--}$mice made significantly fewer contacts with the novel object $(0.45 \pm 0.10$ contacts/ min, mean $\pm \mathrm{SEM} ; n=15)$ than the WT mice did $(1.13 \pm 0.20$; $n=17)(P<0.01)$. Another interesting finding is that in an 8 -arm radial maze test, Cadps $2^{-/-}$mice showed decreased locomotor 


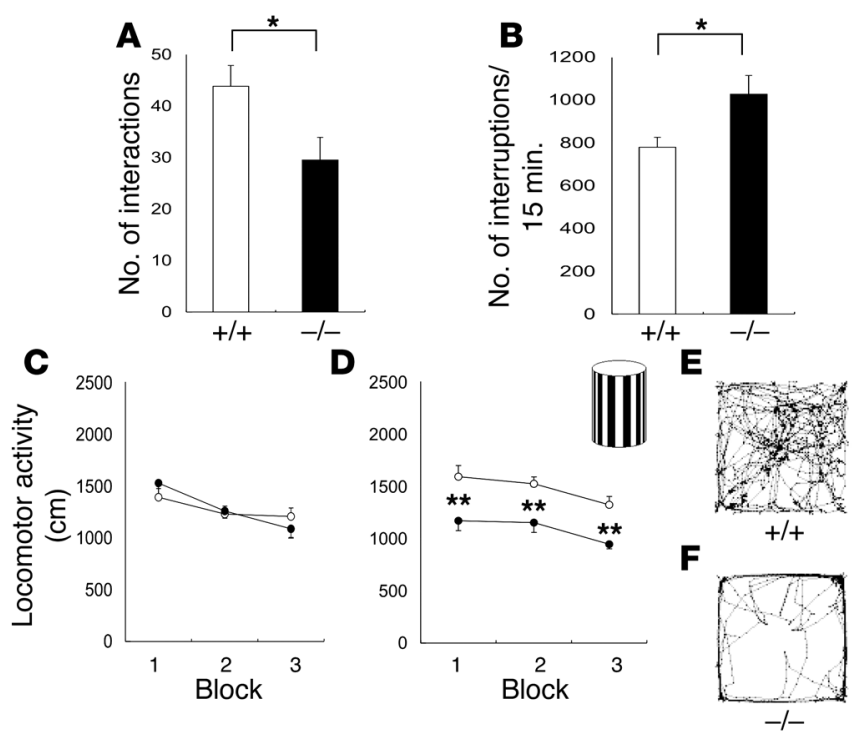

activity $(P<0.05$; Student's $t$ test $)$ and lower arm entries $(P<0.01$; Student's $t$ test) compared with those of WT mice (Supplemental Figure 5; see Discussion).

Fourthly, the Cadps $2^{-/-}$mice had deficits in intrinsic sleep-wake regulation and circadian rhythmicity (Figure $3, \mathrm{~A}-\mathrm{C}$ ). When the circadian rhythm of locomotor activity was recorded under a 12-hour LD cycle, no difference in sleep-wake rhythm was detected between the WT and Cadps2-/- mice (Figure 3, A and B). Under constant dark (DD) conditions, the sleep-wake rhythm of the WT mice shifted to a shorter period than under LD conditions, because the internal circadian period of mice is less than 24 hours (Figure 3, A and C). The Cadps2 $2^{--}$mice, however, exhibited a longer intrinsic rhythmicity under DD conditions $(P<0.01$; Student's $t$ test; Figure 3, B and C).

In addition, maternal neglect of newborns is a striking feature of Cadps $2^{-/-}$mothers. Because maternal care is an important social behavior, the nurturing of newborns by their mothers was monitored. In many cages, the newborns of a Cadps $2^{-1-}$ dam and WT sire rarely survived beyond P1 (Figure 4A). This phenomenon was independent of litter size and almost always occurred in an allor-none manner in each cage. At 2 days after birth, the ratio of extinction cages to survival cages was 1:14 for WT dams, 3:11 for Cadps $2^{+/-}$dams, and 12:7 for Cadps2-/- dams (Figure 4B), indicating a high extinction rate for pups born from Cadps2-/- dams. In this test, heterozygote mice showed roughly intermediate phenotypes between Cadps2-/- and WT mice (Figure 4, A and B). A mildly affected level in heterozygotes was also observed in the 8-arm radial maze test (Supplemental Figure 5). These phenomena are

\section{Figure 3}

An abnormal sleep/wake rhythm of Cadps2-/- mice. (A and B) Sleep/ wake rhythms of locomotor activity under free wheel-running conditions. Representative activity traces of WT (A) and Cadps2-/- (B) mice for 7 days of LD cycle and 14 days of constant darkness (DD) are represented as relative deflections from the horizontal line. Actograms are double-plotted over a 48-hour period. Time is expressed in a 24-hour cycle on the top of $\mathbf{A}$ and $\mathbf{B}$. (C) The circadian period (h) calculated by a $\chi^{2}$ periodogram of $C a d p s 2^{-/-}$mice (black bar; $n=9$ ) and WT littermates (open bar; $n=8$ ). The error bars indicate the SEM. ${ }^{\star *} P<0.01$, Student's $t$ test.

\section{Figure 2}

Autistic-like behavior of Cadps2-/- mice. (A) Number of reciprocal interactions of Cadps2-/- mouse pairs (black bar; $n=12$ ) and of WT littermate pairs (white bar; $n=12$ ) for 20 minutes. (B) Locomotor activity in home cages. After habituation to a fresh cage for 24 hours, the locomotor activity of Cadps2 $2^{-1-}$ mice (black bar; $n=9$ ) and WT littermates (white bar; $n=9$ ) was measured for 6 days (12-hour LD cycle [LD]). The number of photobeam interruptions per 15 minutes is shown in the $y$ axis. (C-F) Horizontal locomotor activity of Cadps2-/mice (filled circles; $n=15$ ) and of WT littermates (open circles; $n=17$ ) in an open field is shown in 3 blocks ( 5 minutes each) in the absence (C) or presence (D) of the novel object shown in the upper right of D. Representative movement traces of WT and Cadps2 $2^{--}$are shown in $\mathbf{E}$ and $\mathbf{F}$, respectively. Error bars indicate the SEM. ${ }^{\star} P<0.05$; ${ }^{* *} P<0.01$, by Student's $t$ test.

probably a reflection of the decrease in CADPS2 protein levels in heterozygote mice (Figure 1D). We then performed cross-fostering experiments to determine whether the primary deficit resided within the Cadps2-/- dams or their pups. The results showed that pups born to control WT dams and cross-fostered to Cadps2-/dams tended to fail to survive, whereas many pups born to Cadps2 $2^{-1-}$ dams and cross-fostered to WT dams survived (Supplemental Figure 6). These results indicate that Cadps $2^{-/-}$mothers have defects in maternal behavior.

The brains of Cadps $2^{-/-}$mice also exhibited several abnormal cell phenotypes. CADPS 1 and CADPS 2 proteins are complementarily distributed in many subregions of the mouse neocortex and hippocampus (11). At P8, CADPS2 immunoreactivity in the WT mouse neocortex was predominantly localized in pyramidal cells in cortical layer V (Figure 5A) and was colocalized with BDNF immunoreactivity (Figure 5, B and C). Underdevelopment of GABAergic interneurons is related to autism (23), and differentiation of a subset of neocortical parvalbumin-positive GABAergic neurons is regulated by BDNF (24). At P17, there were significantly fewer parvalbumin-positive interneurons in the Cadps $2^{-/-}$mouse neocortex (Figure 5E) than in the WT neocortex (Figure 5, D and H), which had a similar phenotype to that of $B D N F^{-/-}$mice (24). On

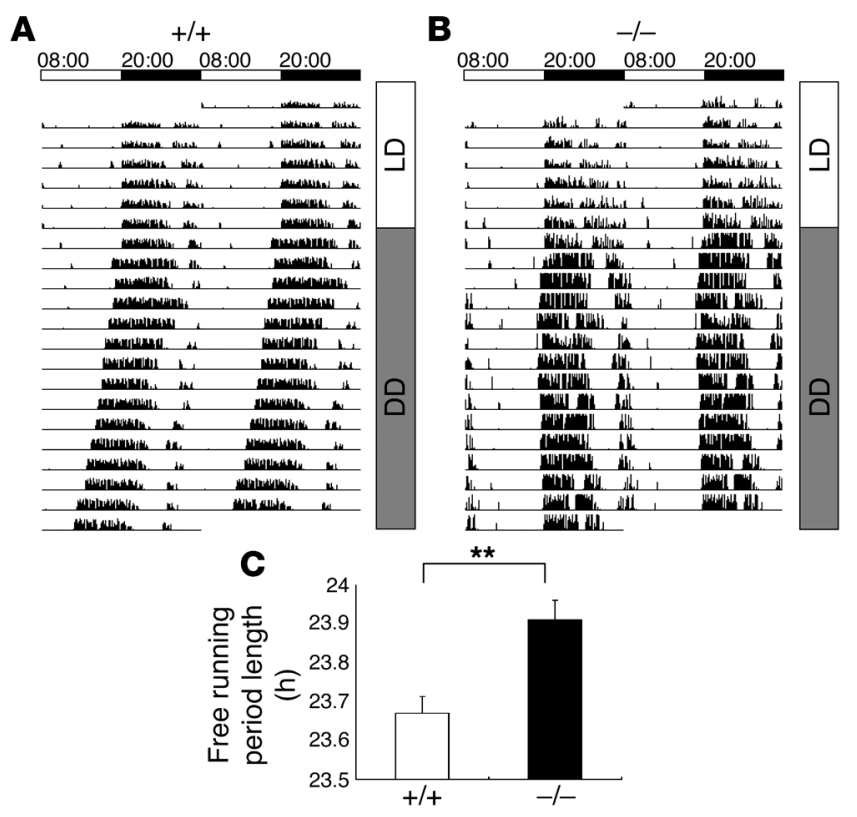


A

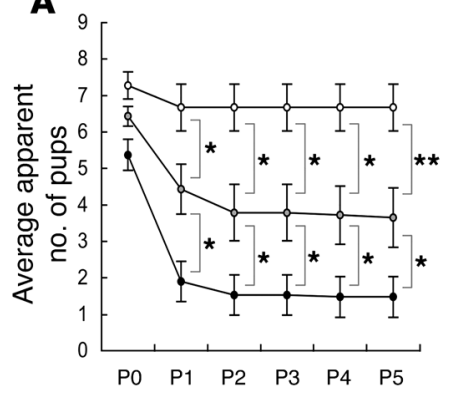

B

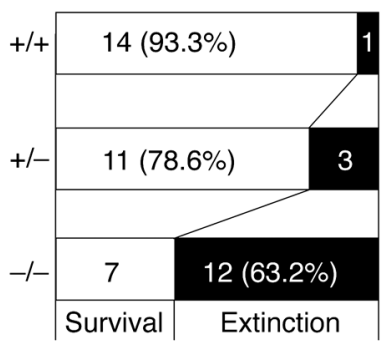

Figure 4

Maternal neglect of newborns by Cadps2 $2^{-/-}$mothers. (A) Survival of pups born to virgin Cadps2--- (closed circles; $n=19$ mothers), Cadps2+- (gray circles; $n=14$ mothers), and WT (open circles; $n=15$ mothers) females mated with WT males. Shown is mean \pm SEM. ${ }^{*} P<0.05 ;{ }^{* *} P<0.01$, Mann-Whitney $U$ test. (B) Number of cages that contained live pups (white bars) versus number of pup extinction cages (black bars) 2 days after birth.

the other hand, there was no significant difference in the number of calbindin-positive neurons at P17 in the neocortex between WT $\left(133 \pm 15\right.$ cells $/ \mathrm{mm}^{2}$, mean $\left.\pm \mathrm{SD}\right)$ and Cadps $2^{2-}$ mice $\left(120 \pm 10\right.$ cells $/ \mathrm{mm}^{2}$, mean $\pm \mathrm{SD}$ ). The decrease in the number of parvalbuminpositive interneurons in the Cadps2 $2^{-/}$mouse neocortex at P17 could be rescued by BDNF injection at P5 (see Methods; Figure 5, G and H), but not by vehicle injection (Figure 5, $\mathrm{F}$ and $\mathrm{H}$ ). However, the number of calbindin-positive neurons was not significantly changed in Cadps $2^{-/-}$mice following either PBS injection $\left(116 \pm 15\right.$ cells $/ \mathrm{mm}^{2}$, mean \pm SD) or BDNFinjection $\left(119 \pm 24\right.$ cells $/ \mathrm{mm}^{2}$, mean \pm SD). Moreover, the levels of BDNF released into the media of Cadps $2^{-/-}$mouse neocortical cell cultures at 21 days in vitro (DIV) were markedly decreased to approximately $22.1 \%$ of the levels observed in the media of WT cell cultures (Figure 5I). On the other hand, there was no significant difference in the levels of neurotrophin 3 (NT-3) or nerve growth factor between WT and Cadps2 $2^{--}$culture medium at 21 DIV (NT-3: $10.3 \pm 0.937 \mathrm{pg} \bullet \mathrm{mm}^{2} / \mu \mathrm{l}$

in WT culture versus $11.7 \pm 1.078 \mathrm{pg} \bullet \mathrm{mm}^{2} / \mu$ in Cadps $2^{-/-}$culture, mean \pm SD; NGF: $395.3 \pm 70.8 \mathrm{pg} \bullet \mathrm{mm}^{2} / \mu \mathrm{l}$ in WT culture versus $304.4 \pm 71.8 \mathrm{pg} \bullet \mathrm{mm}^{2} / \mu \mathrm{l}$ in Cadps2 $-/$ - culture, mean $\pm \mathrm{SD}$ ).

In the WT mouse hippocampus at P8, CADPS2 immunoreactivity was localized predominantly in the stratum lucidum of hippocampal subfield $\mathrm{CA} 3$, where the axons of dentate granule cells (mossy fibers) are projected (Figure 6, A and D), and was colocalized with BDNF immunoreactivity (Figure 6, B, C, E, and F). Immunoreactivity of CADPS2 was not only colocalized with that of the presynaptic proteins chromogranin A and synaptophysin in the CA3 stratum lucidum but was also merged with that of Tau, an axonal marker, in hippocampal primary cultures (Supplemental Figure 7), suggesting a localization of CADPS2 in the axon terminal. By contrast, in the CA1 region, CADPS2 immunoreactive puncta were not merged with those of BDNF (Supplemental Figure 8). At P17, the Cadps2-/- hippocampus showed a reduction in the number of parvalbumin-positive interneurons in comparison with the WT hippocampus (Figure 6, G, H, and K); this reduction was also reported in $B D N F^{-/-}$mice (24). The decrease in the number of parvalbumin-positive interneurons in the Cadps2 $2^{-/}$hippocampus at P17 could be rescued by BDNF injection at P5 to a level comparable with that of the WT hippocampus (Figure 6, J and K).

A decreased Purkinje cell survival rate is a characteristic of the cerebellum in autism (25). CADPS2 is abundant on or near vesicular structures containing BDNF in the parallel fiber terminals of presynaptic granule cells, and overexpression of exogenous CADPS2 in cerebellar primary cultures promotes the survival of postsynaptic Purkinje cells (10). There were considerably fewer calbindin-positive Purkinje cells in cell cultures of Cadps2-- cerebellum, the number being approximately $41 \%$ of that in WT cultures (Figure 7A). However, the number of neurons positive for the neuronal differentiation marker MAP2ab, mostly granule cells, was almost the same as in WT cultures (Figure 7B), indicating that Purkinje cell viability is vulnerable to the loss of CADPS2. To verify whether increased Purkinje cell death was caused by decreased BDNF levels in Cadps2 ${ }^{-/}$cerebellar cultures, we carried

\section{Figure 5}

Abnormal immunohistochemical findings in the Cadps2 $2^{-/}$mouse neocortex. (A-C) Sagittal sections of the P8 motor cortex immunostained for CADPS2 (green in A) and BDNF (red in B). A merged image is shown in C. I, II/III, IV, V, and VI represent cortical layers. Scale bars: $100 \mu \mathrm{m}$. (D-G) Sagittal sections of WT (D) and Cadps2-/- (E-G) P17 motor cortex immunostained for parvalbumin. (F and $\mathbf{G}$ ) Sections were prepared from $\mathrm{P} 17$ Cadps2 $2^{-/-}$mice 12 days after an icv injection of either vehicle (veh inj) (F) or BDNF (BDNF inj) (G). Scale bars: $200 \mu \mathrm{m}$. (H) Cell density of parvalbumin-positive neurons in the $\mathrm{P} 17$ motor cortex. The error bars indicate SD. (I) BDNF release activity in WT (white bars) and Cadps2-/- (black bars) neocortical cultures was evaluated at 21 DIV by measuring the amounts of BDNF spontaneously secreted into the culture medium over the course of 21 days. Activity is indicated in BDNF concentration (pg/ $\mu \mathrm{l})$ normalized to cell density $\left(/ \mathrm{mm}^{2}\right)$. Average values obtained from 6 independent experiments are shown. The error bars indicate SD. ${ }^{* *} P<0.01$, Student's $t$ test.
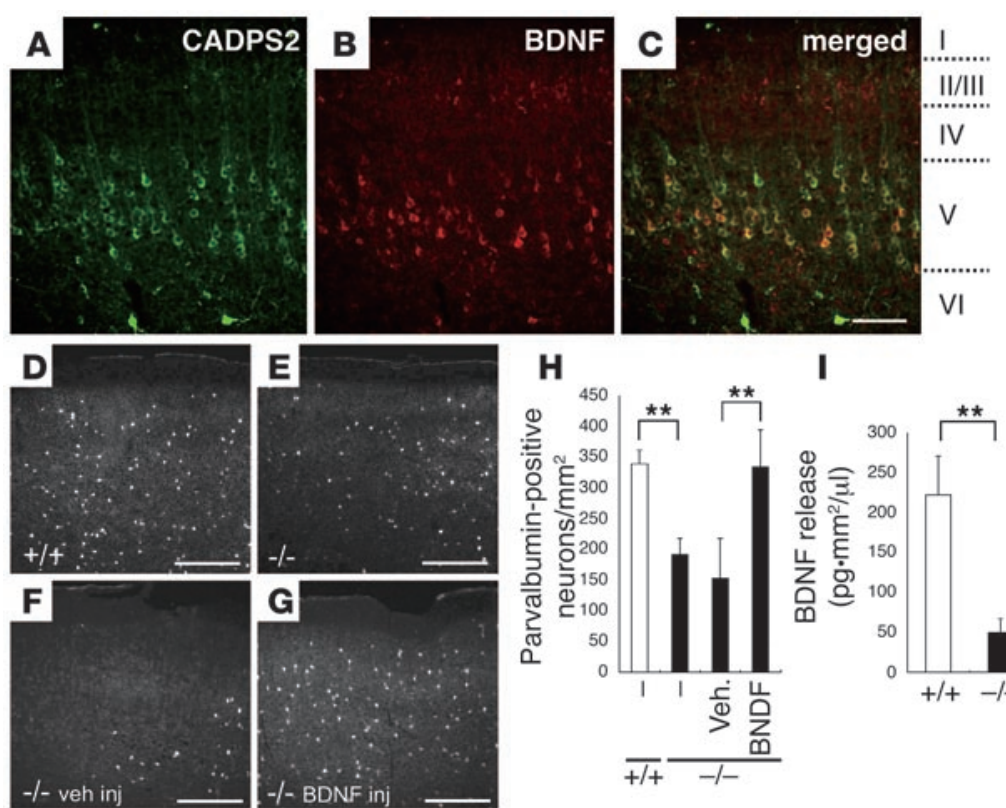

I

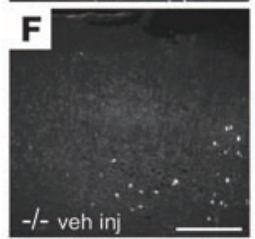

- BDNF in 

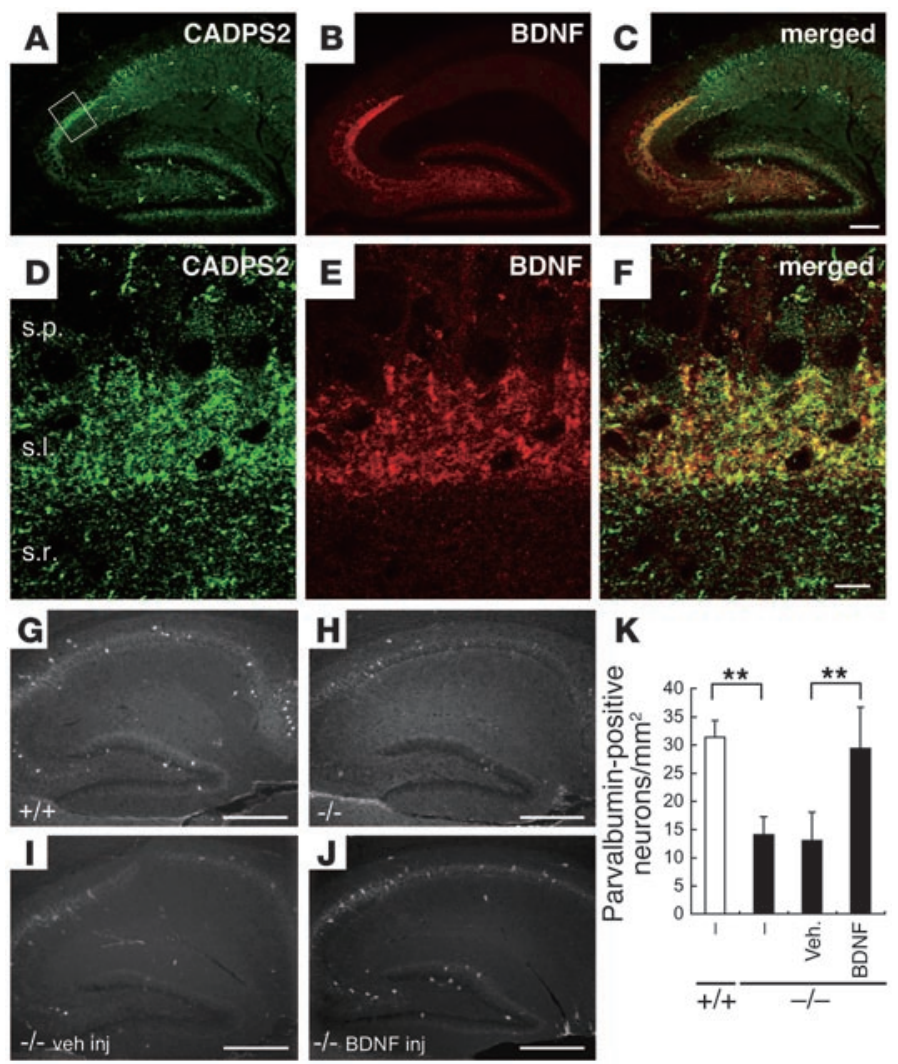

\section{Figure 6}

Abnormal immunohistochemical findings in the Cadps2 $2^{-/-}$mouse hippocampus. (A-C) Sagittal sections of the P8 hippocampus immunostained for CADPS2 (green in A) and BDNF (red in B). A merged image is shown in C. Scale bar: $200 \mu \mathrm{m}$. (D-F) Higher magnification of the region shown by the square in A. s.p., stratum pyramidale; s.I., stratum lucidum; s.r., stratum radiatum. Scale bar: $10 \mu \mathrm{m}$. (G-J) Sagittal sections of WT (G) and Cadps2 $2^{-/-}(\mathbf{H}-\mathbf{J})$ P17 hippocampus immunostained for parvalbumin. (I and J) Sections were prepared from P17 Cadps2-/- mice 12 days after an icv injection of either vehicle (I) or BDNF (J). Scale bars: $500 \mu \mathrm{m}$. (K) Cell density of parvalbumin-positive neurons in the $\mathrm{P} 17$ hippocampus. The error bars indicate SD. ${ }^{* *} P<0.01$, Student's $t$ test. out a cell rescue assay in the presence of exogenous BDNF. By culturing cells in culture media containing BDNF $(10 \mathrm{ng} / \mathrm{ml})$, the survival rate of Cadps $2^{-/}$Purkinje cells was significantly increased in comparison with cultures containing no BDNF or heat-inactivated $\mathrm{BDNF}$ (at $100^{\circ} \mathrm{C}$ for 15 minutes, $10 \mathrm{ng} / \mathrm{ml}$ ) (Figure $7 \mathrm{~A}$ ). In addition, a reduction in the number of calbindin-positive Purkinje cells was verified in sections of the P28 cerebellum (131.3 \pm 7.7 cells $/ \mathrm{mm}^{2}$ in WT versus $104.4 \pm 10.3$ cells $/ \mathrm{mm}^{2}$ in Cadps $2^{-/-}$, mean $\pm \mathrm{SD}$; $<0.01$; Student's $t$ test).

To investigate the role of CADPS2 in neurotrophin secretion, we examined constitutive and regulated BDNF release from primary cell cultures of Cadps $2^{-/-}$mice. As shown in Figure 5I, spontaneous BDNF release from neocortical dissociated cultures at 21 DIV (constitutive release plus spontaneous activity-dependent release) was significantly reduced relative to that of WT cultures. However, BDNF was undetectable in the culture media after $50 \mathrm{mM} \mathrm{KCl}$ stimulation for 15 and 30 minutes (activity dependent, regulated release) in both WT and Cadps $2^{-/-}$neocortical cultures (data not shown). Similarly, the endogenous BDNF released after $\mathrm{KCl}$ stimulation was hardly detected in media of cerebellar

\section{Figure 7}

Increased cell death of Cadps2-- Purkinje cells. (A and B) Cell density of calbindin-positive (A) and MAP2ab-positive (B) neurons in primary dissociated cultures (8 DIV) of WT (white bars) and Cadps2 $2^{--}$(black bars) cerebella. Cerebellar cultures were grown in the absence or presence of the BDNF (10 ng/ml). Heat-inactivated BDNF (HI-BDNF; $10 \mathrm{ng} / \mathrm{ml}$; $100^{\circ} \mathrm{C}, 15$ minutes) was also used. The medium was changed at 4 DIV with readdition of BDNF or HI-BDNF. The error bars indicate SD. ${ }^{\star *} P<0.01$, Student's $t$ test. dissociated cultures at 7, 14, and 21 DIV, which was probably due to a very low amount of endogenous BDNF released from primary cultured neurons as well as insufficient sensitivity of our ELISA system, as previously described (10). We therefore exogenously overexpressed BDNF in cerebellar cultures at 5 DIV using the recombinant adenoviral vector (10). In this system, granule cells, which are the predominant cell type expressing CADPS2 in mouse cerebellum $(10,11)$, were preferentially infected with the adenovirus vector (26). At 48 hours after infection with the adenovirus vector, cultures expressing either BDNF-GFP (Ad-BDNF-GFP) or GFP alone (Ad-GFP) at 7 DIV were treated for 15 minutes with medium containing $10 \mu \mathrm{M}$ NBQX (AMPA receptor inhibitor) plus $1 \mu \mathrm{M} \operatorname{TTX}\left(\mathrm{Na}^{+}\right.$channel inhibitor) to
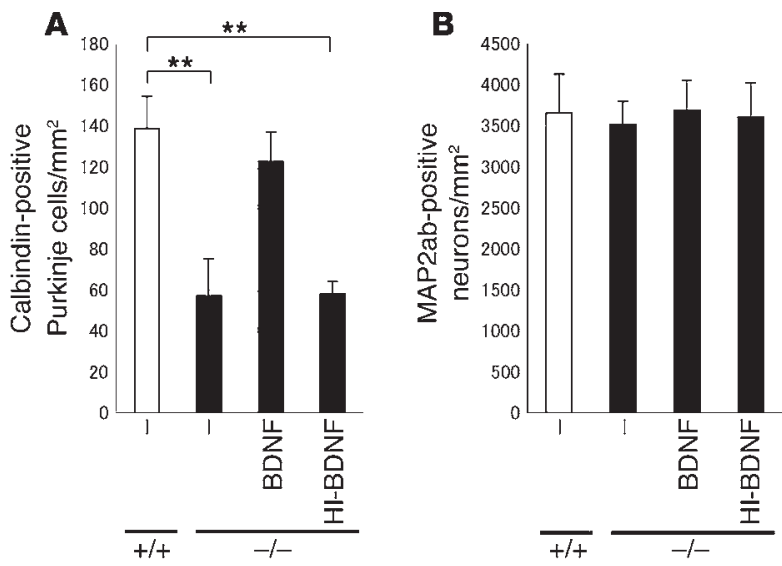


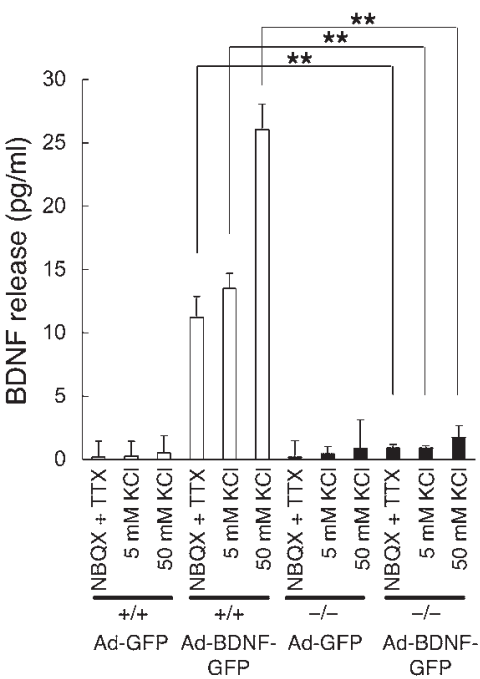

inhibit membrane excitability, $5 \mathrm{mM} \mathrm{KCl}$ as a control, or $50 \mathrm{mM}$ $\mathrm{KCl}$ to induce membrane depolarization. WT cultures overexpressing exogenous BDNF showed high BDNF levels in the NBQX plus TTX and the $5 \mathrm{mM} \mathrm{KCl}$ media (mostly via constitutive release) and even higher levels of BDNF in the $50 \mathrm{mM} \mathrm{KCl}$ medium (via regulated release) compared with control (Ad-GFP) cultures (Figure 8). In contrast, such drastic increases in constitutive and regulated BDNF release levels were not observed

\section{Figure 8}

Decreased release of BDNF from Cadps2-/- cerebellar cultures. WT (white bars) and Cadps2 ${ }^{-/-}$(black bars) cerebellar cultures were infected with the Ad-GFP and Ad-BDNF-GFP at 5 DIV. ELISA of BDNF levels released into the conditioned media from the cultures at 7 DIV with NBQX plus TTX, $5 \mathrm{mM} \mathrm{KCl}$, and $50 \mathrm{mM} \mathrm{KCl}$ stimulation for 15 minutes. Average values obtained from 3 independent experiments are shown. There was no significant difference in MAP2ab-positive cell density and cellular BDNF contents between the WT and Cadps2-/- cultures infected with Ad-BDNF-GFP (data not shown). The error bars indicate SD. ${ }^{\star \star} P<0.01$, Student's $t$ test.

in Ad-BDNF-GFP-infected Cadps2 $2^{-/-}$cultures (Figure 8). These results suggest that loss of CADPS2 affects both constitutive and regulated $\mathrm{BDNF}$ release from cerebellar dissociated cultures.

We next examined the expression of CADPS 2 in autistic patients. Since both CADPS2 protein and CADPS1 protein are expressed in the histamine-positive basophilic leukocytes of healthy subjects (Supplemental Figure 9), we analyzed RNA samples from peripheral blood by RT-PCR with 8 primer sets that covered almost the entire CADPS2 protein-coding region. It was notable that the amplicons derived from the primer set for exons 1-5 included a shorter band (328 bp) in addition to the band of expected size (661 bp) in 3 autistic patients (Figure 9A). Moreover, only a shorter band (328 bp) was detected in 1 patient (Figure 9A). Cloning and sequencing analysis showed that the shorter, 328-bp band lacked the complete sequence of exon 3 (Figure 9C), suggesting an aberrant alternative splicing of CADPS2 mRNA. In contrast, no exon

A

Autistic patients

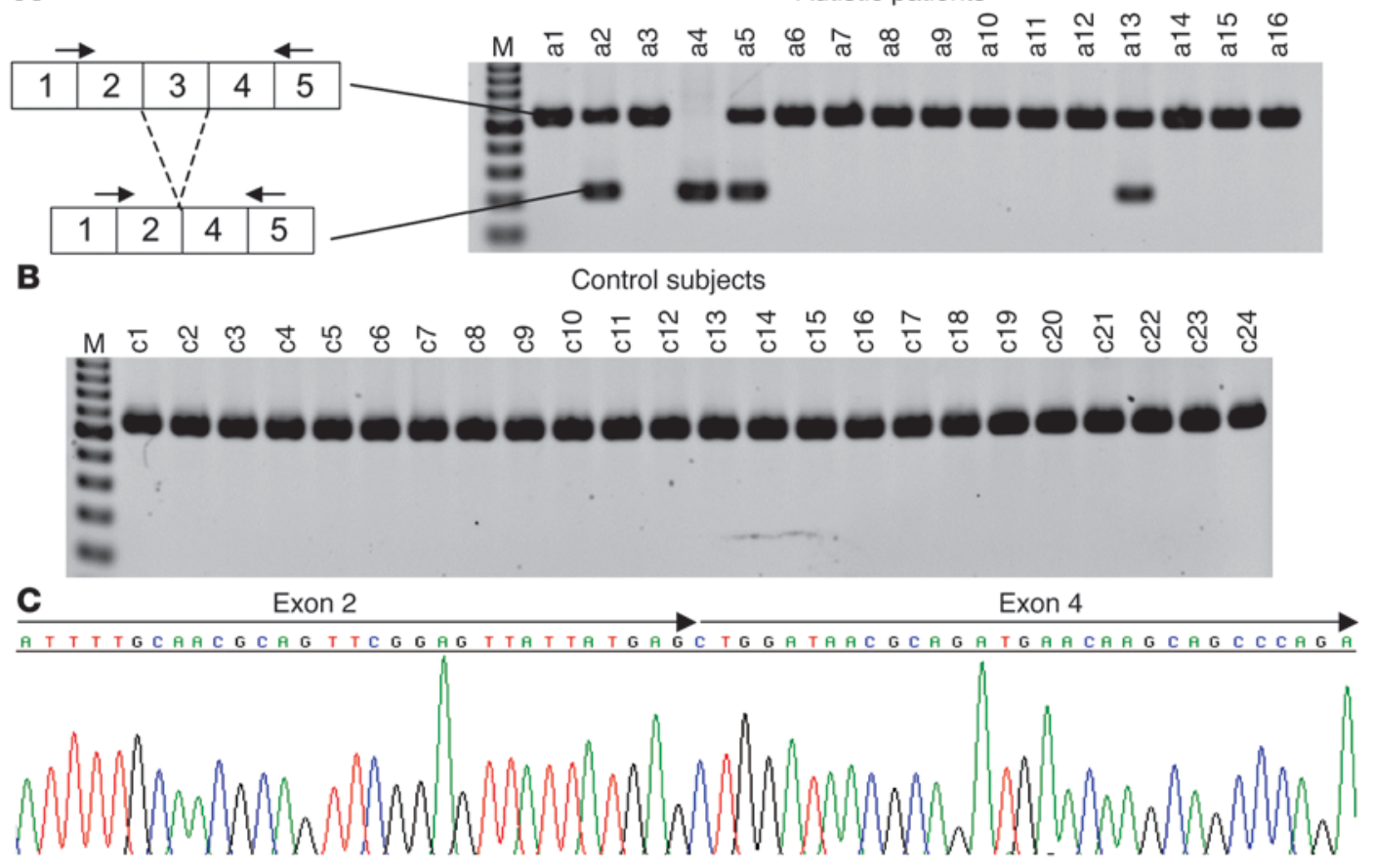

Figure 9

Aberrant CADPS2 splicing in autistic patients. (A and B) RT-PCR analysis of CADPS2 mRNA in blood from autistic patients (a1-a16) (A) and healthy control subjects (c1-c24) (B). A single major band (661 bp) is apparent in the control individuals. An additional band (381 bp) that resulted from skipping of exon 3 was detected in patients a2, a4, a5, and a13. The primers used are indicated on the corresponding exons by arrows. $\mathrm{M}$, 100-bp ladder molecular size marker (from $200 \mathrm{bp}$ at the bottom to $1000 \mathrm{bp}$ at the top). (C) Sequencing pattern of the aberrant RT-PCR product from patient a4. The arrows indicate exon 2-exon 4 in the frame without exon 3. The same results were obtained in 3 other patients (a2, a5, and a13). 
A

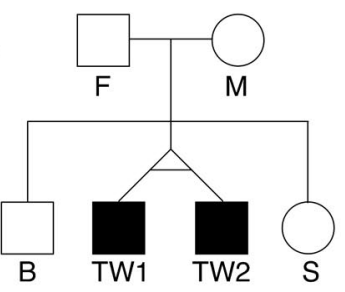

B
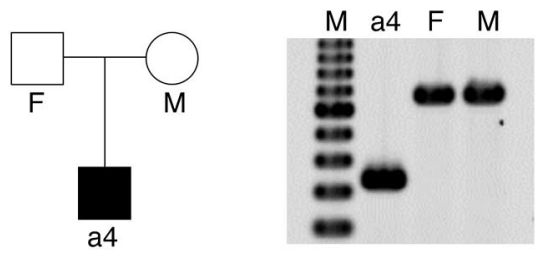

Figure 10

Expression of exon 3-skipped CADPS2 mRNA in 2 pedigrees that include autistic patients. (A and B) Pedigree structures (left) and patterns of agarose gel electrophoresis on the RT-PCR analysis of CADPS2 mRNA expressed in the blood of each individual (right). (A) Male monozygotic autistic twins (TW1 [autistic patient a5 shown in Figure 9A] and TW2 [autistic patient]) and their family members. A single major band (661 bp) produced by normal alternative splicing is apparent in their father $(\mathrm{F})$; mother $(\mathrm{M})$; older brother $(\mathrm{B})$; and younger sister (S). On the other hand, an additional, 381-bp band produced by exon 3-skipped alternative splicing was expressed in both monozygotic autistic twins. (B) The autistic patient (patient a4 shown in Figure 9A), who expressed only the exon 3-skipped shorter band, and the patient's family members. Similar to the family members in $\mathbf{A}$, a single major band of $661 \mathrm{bp}$ is expressed in the father and mother. M, molecular weight marker of the 100-bp ladder (from 200 bp at the bottom to $1000 \mathrm{bp}$ at the top).

3 skipping was found in any of the 24 healthy control subjects tested (Figure 9B) $(P=0.020$; Fisher's exact probability test). Exon 3 skipping in the mouse counterpart has never been observed in the brain or nonneural tissues of WT mice (data not shown). We next examined the expression of CADPS2 mRNA in the family members of 2 autistic patients (a 4 and a 5 in Figure 9A). Patient a5 in Figure 9A is a monozygotic twin, and the other autistic twin also expresses exon 3-skipped CADPS2 mRNA (Figure 10A). However, neither their parents nor healthy family members (older brother and younger sister) were found to express the exon 3-skipped form (Figure 10A). Similarly, patient a4's family members - a father and mother who are both mentally healthy - do not express the exon 3 -skipped form (Figure 10B). Although the mechanism underlying exon 3 skipping remains elusive, no mutations were found around the splice donor site (within $60 \mathrm{bp}$ downstream), acceptor site (within 80 bp upstream) or branchpoint of intron 2 in the genomic sequences of these patients.

Exon 3 skipping predicts a deletion of 111 aa residues, from 119 to 229 in the human CADPS2 protein (GenBank accession number NP_060424). To investigate the effect of this splicing variant on BDNF-releasing activity, we exogenously expressed full-length (WT) or exon 3-skipped ( $\triangle$ exon3) mouse CADPS2 together with BDNF in PC12 cells. The BDNF released into the culture medium in response to high- $\mathrm{KCl}$ stimulation by cells coexpressing CADPS2(WT) was approximately $200 \%$ relative to that of cells without exogenous CADPS2 expression (Figure 11A), as described previously (10). BDNF release was also increased in cells coexpress- ing CADPS2( $\triangle$ exon3) (Figure $11 \mathrm{~A}$ ), indicating that the exon 3skipped CADPS2 retains BDNF-releasing activity. Moreover, in neocortical primary dissociation cell cultures of Cadps $2^{-/-}$mice, the levels of BDNF released into the culture media at 21 DIV were significantly increased by transfection with either CADPS2(WT) or CADPS2(Aexon3) at 4 DIV (Figure 11B). There was no significant difference in the BDNF content of culture media between CADPS2(WT)-transfected and CADPS2(Aexon3)-transfected cultures. These results indicate that the exon 3-skipped variant has the ability to enhance release activity in in vitro culture systems.

To further investigate the function of exon 3 , we screened for protein candidates that interact with the exon 3 -coded region, by the yeast 2-hybrid method using exon 3-containing residue as bait (Figure 12A). As a result, we isolated 3 candidate genes, coding E1A binding protein p400 (Ep400, also known as mammalian Domino [mDomino]) (27), RAN binding protein 9 (Ranbp9, also known as RanBPM) (28), and dynactin 1 (Dctn1, also known as p150 (liued) (29). Proteins mDomino and RanBPM were excluded from further scrutiny because of a difference in subcellular localizations, i.e., mDomino in the nucleus (27) versus CADPS2 in the cytoplasm and membrane (30) and because of irreproducibility in coimmunoprecipitation experiments in COS-7 cells coexpressing CADPS2 and RanBPM. Two clones coding residues 951-1281 of p150 Glued were independently isolated. Using lysates of COS-7 cells coexpressing $\mathrm{p} 150^{\text {Glued }}$ and CADPS2 constructs, we confirmed that the N-terminal FLAG-tagged 951-1281 residue of $\mathrm{p} 150^{\text {Glued }}(40 \mathrm{kDa})$ was coimmunoprecipitated with the C-terminal HA-tagged bait region of CADPS2 (data not shown) or C-terminal HA-tagged full-length CADPS2 (Figure 12B). However, no FLAG-p150 Glued (951-1281 aa) was coimmunoprecipitated with exon 3-skipped CADPS2 (Figure 12B). Moreover, endogenous $\mathrm{p} 150^{\text {Glued }}$ was coimmunoprecipitated with the endogenous CADPS2 in lysates of mouse neocortex (Figure 12C), and immunostaining signals for p150 Glued was merged with those for CADPS2 in primary neocortical, hippocampal, and cerebellar cultures (data not shown). These results provide strong evidence for CADPS2-p150 Glued interaction via the region coded by exon 3 .

The protein $\mathrm{p} 150^{\text {Glued }}$ is the most fully characterized subunit of the dynactin complex (29), which is associated with axonal transport $(31,32)$. We investigated the subcellular localization of exon 3skipped CADPS2 protein in the calbindin-positive neurons of neocortical primary cultures. We used MAP2ab immunosignals to identify the axons of calbindin-positive neurons, as reported elsewhere (33). As shown in Figure 13A, expressed full-length CADPS2 protein was distributed in axons (shown as MAP2abnegative and calbindin-positive in Figure 13C), whereas expressed exon 3-skipped CADPS2 protein was localized to dendrites and none were detected in axons (Figure 13, B and D). Similarly, fulllength CADPS2 protein expressed in cerebellar primary cultures was distributed in both MAP2ab-negative axons and MAP2abpositive dendrites (Figure 13E), whereas expressed exon 3skipped CADPS2 protein was localized primarily to MAP2abpositive dendrites but not to axons (Figure 13F). On the other hand, coexpression of exon 3-skipped CADPS2 and GFP-tagged BDNF in Cadps2 $2^{-/}$cerebellar cultures resulted in the localization of BDNF-GFP in axons, with exon 3-skipped CADPS2 being absent from axons (data not shown). This result suggests that the axonal transport of BDNF is not affected by the absence of CADPS2 protein at axons. 
A

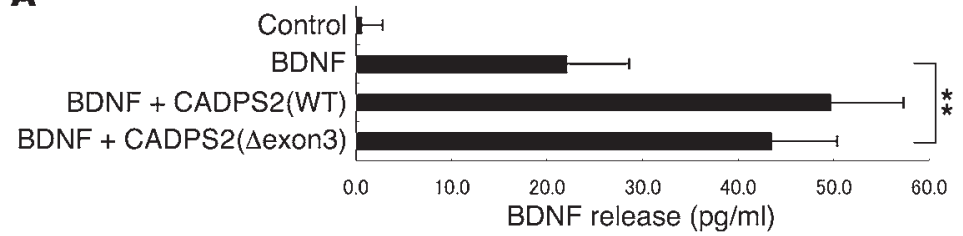

B

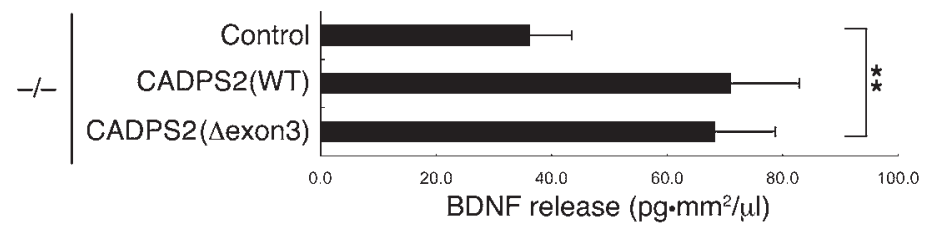

Figure 11

Functional analyses of CADPS2 exon 3. (A) BDNF release induced by $50 \mathrm{mM}$ $\mathrm{KCl}$ in PC12 cells transfected with CADPS2(WT) or CADPS2( $\Delta$ exon3) together with a BDNF expression plasmid. Average values obtained from 4 independent experiments are shown. (B) BDNF release activity in $\mathrm{Cadps}^{-/-}$neocortical cultures transfected with control vector, CADPS2(WT), or CADPS2( $\triangle$ exon3) using the calcium phosphate method at 4 DIV was evaluated at 21 DIV by measuring the amounts of BDNF spontaneously secreted into the culture medium over the course of the previous 17 days. Activity is indicated in BDNF concentration (pg/ul) normalized to cell density $\left(/ \mathrm{mm}^{2}\right)$. Average values obtained from 4 independent experiments are shown. The error bars indicate SD. ${ }^{\star \star} P<0.01$, Student's $t$ test.

Finally, we examined nonsynonymous SNPs over the entire CADPS2-coding sequence. A previous study showed that no patient-specific nonsynonymous (missense) SNPs were identified in the CADPS2 gene from 90 autistic individuals, using the heteroduplex formation-detection method (9). However, our sequence analyses of 252 white autistic patients revealed 7 nonsynonymous SNPs in the CADPS2 gene that we believe to be novel (Supplemental Table 2 and Supplemental Figure 11). By contrast, these SNPs were not observed in 218 biologically unrelated white subjects from cohorts recruited as bipolar disorder pedigrees $(34,35)$, as shown in Supplemental Table 2. In addition, they have not been reported in healthy human subjects. These results suggest the possibility that the 7 SNPs identified within the CADPS2-coding sequence are associated with some autistic patients, although whether these SNPs have any relation to the specific phenotypes of autism remains elusive.

\section{Discussion}

Our knockout mouse study shows that the loss of CADPS2 function in mice causes impairments in behavioral phenotypes (social interaction, home cage activity, response to novel environment, circadian rhythm, and maternal behavior) that are reminiscent of the impairments that characterize autistic patients. Analyses of the cellular phenotypes of Cadps2-knockout mice revealed that CADPS2 is critical for BDNF secretion from neocortical and cerebellar neurons, the differentiation of neocortical and hippocampal interneurons (deficits in which can be rescued by BDNF injection), and the survival of cerebellar Purkinje cells. Moreover, we found that CADPS2 mRNA from the blood of some autistic patients is aberrantly spliced, resulting in a loss of exon 3, which has been shown to encode the domain that binds to $150^{\text {Glued. }}$. We also showed that exon 3-skipped CADPS2 protein has almost normal BDNF releasing activity but is not properly transported into the axons of neocortical and cerebellar neurons. These results suggest that, while BDNF localization at the axon terminal is not altered by the exon 3 skipping of CADPS2, the loss or relative decrease of BDNF release from the axon terminal is caused by the impaired translocation of CADPS 2 to the axon terminal (Supplemental Figure 10).

CADPS2 plays an important role in the BDNF secretion. CADPS was previously implicated in the regulation of dense-core vesicle secretion from endocrine and neuroendocrine cells through the activation of the phosphatidyl inositol 4,5-bisphosphate-dependent and $\mathrm{Ca}^{2+}$ dependent priming step (36-39). On the other hand, a recent study of Cadps1-knockout mice, which die shortly after birth, suggested a role for CADPS1 in the loading of catecholamines into secretory vesicles (40). Therefore, there is a possibility that CAPS/CADPS family proteins are involved in multiple steps in the secretory pathway, although their underlying molecular mechanisms remain elusive. Our previous study suggested the involvement of CADPS2 in the release of BDNF and NT-3 from cerebellar granule cells (10). The present study confirms the indispensable role of CADPS2 in BDNF secretion by showing that CADPS2 deficiency causes impairment of BDNF release from neocortical and cerebellar primary cell cultures. It was of interest that the deficit of BDNF release was observed in both regulated and constitutive processes in cerebellar cultures. These data suggest that CADPS2 is a component involved in a few steps of the secretion pathway, including loading and exocytosis steps, and that CADPS2 is likely involved in a common mechanism of the regulatory and constitutive secretion processes. On the other hand, an immunolocalization study indicated that the distribution patterns of CADPS2 and BDNF completely overlap in many brain areas but overlap incompletely in other areas (11). Thus, although CADPS2 is required for BDNF release from many brain areas, $\mathrm{BDNF}$ release from other areas, including the hippocampal CA1 region, might be attributed to the function of another CAPS/ CADPS family protein, CADPS1. The differential immunolocalization of CADPS2 and BDNF in some brain areas (11) suggests that CADPS2 is involved in the release of secretory substances other than BDNF in these areas.

Abnormal behavioral phenotypes characteristic of Cadps2-knockout mice. Cadps2-knockout mice not only have a normal locomotion ability but also show normal basic sensory functions such as vision, olfaction, and audition. In a probe test of Morris water maze learning, however, Cadps2-knockout mice showed impaired spatial memory. It was shown that the probe test, for example, a hidden platform test, reflects spatial learning better than measures of escape latencies during training (41). Taking these results together, some part of cognitive function (that is required for Morris water maze learning) appears to be impaired in Cadps2-knockout mice.

Novel stimuli, such as unfamiliar environments or objects, are theorized to create conflict in rodents by concurrently evoking both approach and avoidance behaviors (22). Approach behavior, or "exploration", reflects an animal's tendency to explore novel stimuli or environments, whereas avoidance behavior, or "anxietyrelated behavior," is thought to reflect an animal's fear of novelty. Cadps $2^{-/-}$mice showed decreased locomotor activity compared with WT mice (Figure 2D) when placed in an open field containing a novel object, as shown in dopamine D4 receptor-knockout mice (42). Moreover, Cadps2 $2^{--}$mice made significantly fewer contacts 


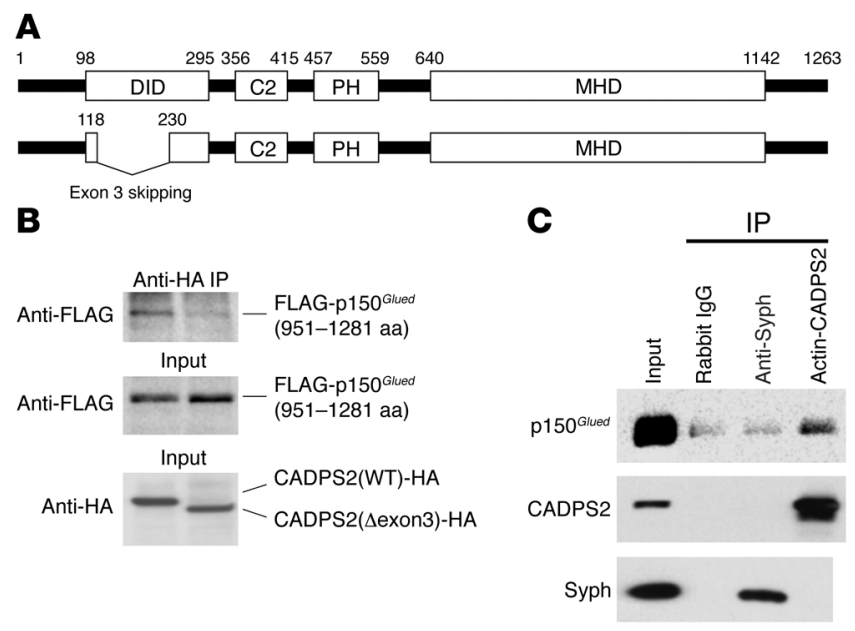

Figure 12

Interacting protein of CADPS2 exon 3. (A) Schematic depiction of human CADPS2 protein (GenBank accession number NP_060424). The C2- and pleckstrin homology (PH)-like domain, Munc13-1-homologous domain (MHD), and p150 Glued (also known as dynactin 1) -interacting domain (DID) used as bait are shown. Exon 3 skipping leads to a deletion of 111 aa residues (from 119 to 229). (B) Coimmunoprecipitation experiments using lysates of COS-7 cells coexpressing p150 Glued 951-1281 residues and CADPS2 constructs tagged with $\mathrm{N}$-terminal FLAG and C-terminal HA epitopes, respectively. Coimmunoprecipitates with anti-HA antibody to CADPS2 constructs were blotted with antiFLAG antibody (upper panel). Input lysates were blotted with anti-FLAG antibody (middle panel) and anti-HA antibody (lower panel). (C) The endogenous $\mathrm{p} 150$ Glued was coimmunoprecipitated with the endogenous CADPS2 in P8 mouse neocortex extracts but not with endogenous synaptophysin (Syph). The blots were immunostained for p150 Glued (upper panel), CADPS2 (middle panel), and synaptophysin (lower panel).

with the novel object. These results indicate that Cadps $2^{-/-}$mice tend to show augmented anxiety or reduced environmental exploration in a novel environment, as was also the case in dopamine D4 receptor-knockout mice (42). Interestingly, in an 8-arm radial maze test, Cadps2 $2^{-/}$mice showed decreased locomotor activity and lower arm entries relative to WT mice (Supplemental Figure 5). Correspondingly, the selective dopamine D2 receptor agonist, LY-171555, has been found to cause decreased locomotion in an 8-arm radial maze test (43) along with hyperactivity in the home cages (44). As the CAPS/CADPS family proteins interact with a dopamine D2 receptor (45), the phenotypes observed in the present study might be associated with the dopamine pathway. Similar behaviors in unfamiliar environments are observed in patients with autism $(1,2,19)$.

$B D N F$ release activity of exon 3-skipped CADPS2. We found that some autistic patients express an aberrantly spliced, exon 3 skipped variant of CADPS2 mRNA in their blood. It is a notable fact that this variant form was detected in both autistic monozygotic twins. Exon 3-skipped CADPS2 proteins exogenously expressed in PC12 cells and primary cultured cerebellar and neocortical neurons had a BDNF-releasing activity similar to that of WT CADPS2. From these in vitro expression data, we suggest that the levels of BDNF in the blood of autistic patients expressing exon 3-skipped CADPS2 might be unchanged in comparison with those from healthy control subjects or patients expressing only the WT CADPS2. On the other hand, there have been several reports showing different pathological results regarding BDNF levels in the sera of autistic patients, including increased levels (46-48), no difference (49), and decreased levels (50). As for this point, the origin of blood BDNF is still an open question; that is, it remains unknown from which cell types and by which releasing mechanisms BDNF is released (constitutive versus regulated, CADPS1 mediated versus CADPS2 mediated, etc.). Therefore, at present, we cannot state anything conclusive about the association of BDNF levels in the sera with autism.

Disturbance in subcellular localization of exon 3-skipped CADPS2. We have shown here that the aberrant exon 3 skipping in CADPS2 deletes the dynactin-binding domain of the protein product. Our previous study indicated that in the cerebellum, CADPS2 protein accumulates primarily in the presynaptic terminals of granule cells connecting Purkinje cell spines (10). CADPS2-mediated BDNF release seems to trans-synaptically act on Purkinje cells and promote their survival and differentiation. Exogenously expressed exon 3-skipped CADPS2, however, was not transported to the axons of primary cultured neocortical and cerebellar neurons. This improper subcellular targeting of exon 3-skipped CADPS2 seems to cause a disturbance of localized neurotrophin release in neurons (Supplemental Figure 10). The current data suggest that disturbance in the proper levels of localized BDNF release, which is assumed to be caused by aberrant subcellular targeting of CADPS2, results in the abnormal development of circuit connectivity observed in autism (51).

It remains to be resolved whether expression of exon 3-skipped CADPS2 mRNA is paralleled by production of exon 3-skipped CADPS2 protein in autistic patient cells. We tried to analyze CADPS2 protein by Western blotting in the limited volume of blood samples from patients, but unfortunately we could not detect it even in samples from healthy persons. This is probably due to the very low levels of CADPS2 protein in blood cells being expressed only in basophilic leukocytes, which represent only about $0.5 \%$ of total leukocytes. Recombinant exon 3-skipped CADPS2 protein, however, could be stably expressed in PC12 cells and cultured neocortical and cerebellar neurons, as could WT CADPS2 protein. To correlate the present findings to the phenotypes of autistic patients, it must also be determined whether either mRNA and/or protein of exon 3-skipped CADPS2 is expressed in autistic brain tissues.

Plausible mechanisms underlying the aberrant splicing of CADPS2 $m R N A$ in autistic patients. It is still unclear how the splicing out of exon 3 is specifically caused in some autistic patients. We could not find any nucleotide sequence differences in 3 common splicing cis-elements (the $5^{\prime}$ donor and $3^{\prime}$ acceptor sites and the branch point) of the CADPS2 gene from autistic patients displaying exon 3 skipping. In addition to these authentic cis-elements, some splicing events are regulated by additional cis-elements known as exonic and intronic splicing enhancers/silencers. To further pursue the possibilities pointing to a "cis-hypothesis," we examined exon 3 sequences and could find no change in the patients tested. We also tested whether there were microdeletion(s) in the intron 2 interval $(73,385 \mathrm{bp})$ by quantitative genomic PCR, targeting 6 discrete portions within intron 2 sequences in DNA from the autistic subjects represented in Figure 10 (data not shown). As a result, we could not detect any genomic variants/abnormalities in the autistic DNA. Nonetheless the possibility of a "cis-hypothesis" cannot be ruled out because 1 or more mutations might still be present somewhere in the unexplored genomic region within the CADPS2 genes of these autistic patients. 

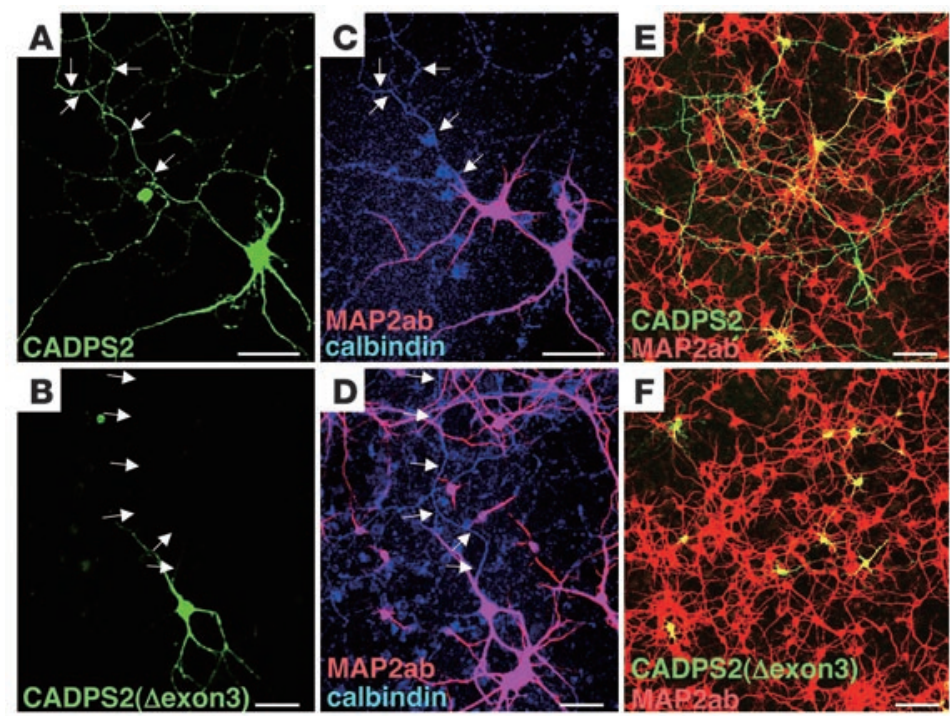

Figure 13

Aberrant distribution patterns of exon 3-skipped CADPS2 protein. (A-D) Subcellular localization of C-terminal HA-tagged CADPS2(WT) (A) and CADPS2( $\triangle$ exon3) protein (B) exogenously expressed in neocortical primary cultures immunostained for HA (green), MAP2ab (red), and calbindin (blue) at 14 DIV. Arrows show the position of MAP2ab-negative and calbindin-positive axons as shown in the same frame (C and $\mathbf{D})$. ( $\mathbf{E}$ and $\mathbf{F})$ Subcellular localization of C-terminal HA-tagged CADPS2(WT) (E) and CADPS2( $\Delta$ exon3) (F) protein exogenously expressed in cerebellar primary cultures immunostained for $\mathrm{HA}$ (green) and MAP2ab (red) at 7 DIV. Scale bars: $50 \mu \mathrm{m}$.

Alternatively, we must also consider the possibility of a "transhypothesis" as an underlying causality - that is, the possibility that there are 1 or more polymorphisms in unknown genes that alters the splicing of CADPS 2 mRNA. This scenario would also predict abnormal splicing of multiple genes in addition to the exon 3 skipping of CADPS2. However, as we do not have any evidence for aberrant splicing in other genes, we need to be cautious in any interpretation of the results of CADPS2 exon 3 skipping based on a trans-hypothesis. In this regard, it seems to be a suggestive example that a mutation in methyl CpG binding protein 2 (MECP2) has been shown to be causative for Rett syndrome, a particular autism spectrum disorder, by affecting the expression level of the $B D N F$ gene $(16,52,53)$; however, MECP2 likely has multiple target genes other than the BDNF gene.

Other potential mechanisms for pathological alternative splicing of CADPS2 in autistic patients would include aberrant epigenetic regulation and de novo mutation. It should be noted, however, that none of these inferred mechanisms explain satisfactorily why the affected individuals in pedigree A show 2 bands, authentic and exon 3-skipped CADPS2 bands (Figure 10), whereas the affected individual in pedigree $\mathrm{B}$ shows only an exon 3-skipped band. These results imply that the causal variants in pedigrees $A$ and $B$ should be different. We can at least exclude a dominant inheritance mechanism of causal variants in both pedigrees, because the parents are unaffected in both pedigrees. Future extensive studies are warranted to expose the precise pathological mechanisms.

Our study also identified 7 nonsynonymous SNPs by analyzing the CADPS2 coding sequence of 252 white autistic patients and 1 case in which CADPS2 mRNA expression was not detected in the blood specimen of that particular autistic patient (see Methods); however, any correlation of these findings with autistic phenotypes remains elusive. We suggest that CADPS2 disturbance due to genetic and/or allelic heterogeneities (e.g., disturbed function, subcellular localization, or expression caused by rare, nonsynonymous mutations, exon 3 skipping, aberrant expressional control, etc.) predisposes individuals to a higher risk for autism, at least in some cases. These phenomena may point to the scenario of "common disease - rare variants" rather than "common disease - common variants," both of which are proposed to contribute to common disease mechanisms. It would be important that future autism studies include an intensive scrutiny of the genetic mechanisms that impact directly and indirectly on the function and structure of CADPS2, as one of the implicated susceptibility genes for autism.

\section{Methods}

Human subjects. The study protocol was approved by the ethics committees of RIKEN Brain Science Institute, Hamamatsu University School of Medicine, and Tokyo Metropolitan Umegaoka Hospital (Tokyo, Japan). RNA samples were obtained from 16 Japanese autistic patients, who were diagnosed according to the DSM-IV (2). Their ages ranged from 13 to 27 years. Informed consent was obtained from all the participants. Control samples were obtained from age-matched healthy Japanese volunteers. DNA samples from 252 white autistic patients were obtained from the Autism Genetic Resource Exchange (AGRE). The diagnostic assessment in the AGRE sample was based on the Autism Diagnostic Interview-Revised (ADI-R) diagnostic test (54). DNA samples from 218 biologically unrelated white subjects from cohorts recruited as bipolar disorder pedigrees $(34,35)$ were also used.

RT-PCR. RNA was extracted from fresh blood with TRIzOL Reagent (Invitrogen) according to the manufacturer's instructions (1 $\mathrm{ml}$ TRIzOL Reagent was added to $100 \mu$ l of blood). RT-PCR reactions were carried out in a 20- $\mu$ l volume in the GeneAmp PCR System 9700 (PerkinElmer). The QIAGEN OneStep RT-PCR kit (QIAGEN) was used according to the manufacturer's instructions. A 50-ng sample of total RNA template was used per reaction. The reverse transcription cycling conditions were 30 minutes at $50^{\circ} \mathrm{C}$ followed by 15 minutes at $95^{\circ} \mathrm{C}$. PCR cycling conditions consisted of 48 cycles of $94^{\circ} \mathrm{C}$ for 30 seconds, $67^{\circ} \mathrm{C}$ for 30 seconds, and $72^{\circ} \mathrm{C}$ for 30 seconds, followed by a final hold at $72^{\circ} \mathrm{C}$ for 5 minutes. The forward primer was 5-GGCAGCAGAAGCTTAACAAACAACAGTTGCAGTTAC-3, and the reverse primer was 5-GGACCACCTTTCGAAACTGGAAGACTTTCC-3 (normal, 661-bp product; exon 3 skipped, 328-bp product). PCR was carried out at least 4 separate times for each sample. PCR products were electrophoresed on $2 \%$ agarose gels with a 100-bp DNA Ladder (catalog no. 15628-050; Invitrogen). All 328-bp products were cloned into the PCR4-TOPO TA cloning vector (Invitrogen), and the exon 3 skipping was confirmed by sequencing. PCR reactions were run at the same time for patients and controls. The RT-PCR product band of CADPS2 mRNA was not detected from 1 autistic patient, although the GAPDH band was detected (data not shown).

Production of the mouse line. A $12-\mathrm{kb}$ genomic fragment containing exon 1 of mouse CADPS2 from C57BL/ 6 mice was used to construct the targeting vectors. For positive selection, the SmaI-SmaI fragment containing the full length of exon 1 was replaced by the Pgk-neo gene cassette flanked by the loxP sites (Figure 1A). For negative selection, the diphtheria toxin A fragment gene cassette was added to the $5^{\prime}$ end of the targeting vector. After transfection of MS12 ES cells (C57BL/6 mouse ES cell line [ref. 55]) by electroporation, targeted clones were screened for G418 resistance and analyzed by Southern blot analysis. Chimeric mice 
were generated by injection of the targeted MS12 ES cells into Balb/c blastocysts and mated with WT C57BL/6J mice to obtain heterozygous mutant mice. All the engineered animals studied were backcrossed onto C57BL/6J for more than 5 generations.

$B D N F$ injection. The icv injection of BDNF in neonatal mouse brains was carried out essentially as described in previous studies $(56,57)$. P5 $\mathrm{C} 57 \mathrm{BL} / 6 \mathrm{~J}$ mice were anesthetized by diethyl ether and then given an icv injection of a $2-\mathrm{ml}$ solution containing either vehicle (PBS, $\mathrm{pH} 7.4$ ) or BDNF ( $5 \mathrm{mg}$ in vehicle) into the left hemisphere. The location of each injection was $2.5 \mathrm{~mm}$ anterior to $\lambda, 0.9 \mathrm{~mm}$ lateral to the midline, and $1.9 \mathrm{~mm}$ below the skull surface. Injections were performed using a Hamilton syringe with a 30 -gauge beveled needle. The needle was withdrawn 2 minutes later and the scalp sutured. Mice were placed in a warm cage until they had fully recovered and then were returned to their home cages with a foster mother mouse. The brains of 8 mice (4 PBS-injected mice and 4 BDNF-injected mice) were fixed at P17 as described below, and 2 sagittal sections of left hemisphere from each mouse (a total of 16 sections) were immunostained.

Immunohistochemistry of brain sections. P8, P17, and P21 C57BL/6J mice anesthetized with diethyl ether were transcardially perfused with PBS and then with $4 \%$ paraformaldehyde in PBS. The brains were dissected, postfixed in $4 \%$ paraformaldehyde at $4^{\circ} \mathrm{C}$ for 5 hours, and cryoprotected by immersion in $15 \%$ sucrose in PBS overnight at $4^{\circ} \mathrm{C}$. After embedding in Tissue-Tek OCT compound (Sakura Finetechnical Co.), the brains were frozen in dry ice powder and cut into $14-\mu \mathrm{m}$ sagittal sections with a cryostat (Leica CM1850; Leica Microsystems) at $-18^{\circ} \mathrm{C}$. The sections were then air dried for 1 hour and rinsed in PBS 3 times. After blocking with $5 \%$ normal donkey serum (Vector Laboratories) in PBS, the sections were reacted with the primary antibody at $4{ }^{\circ} \mathrm{C}$ overnight, rinsed in PBS, reacted with the secondary antibody at room temperature for 1 hour, and again rinsed in PBS. The immunoreacted sections were mounted with VECTASHIELD Mounting Medium (Vector Laboratories) and examined with an epifluorescence microscope (Eclipse E800; Nikon) equipped with a cooled charge-coupled device camera (SPOT model 1.3.0; Diagnostic Instruments Inc.) or a confocal laser microscope (LSM 510 META; Zeiss).

Immunocytochemistry of fractionated leukocytes. Leukocytes were fractioned as described previously (58). Freshly separated leukocytes that had been smeared on 3-aminopropyltriethoxysilane (APS)-coated glass slides and fixed with acetone for 10 minutes at $4{ }^{\circ} \mathrm{C}$ were used for immunocytochemistry.

PC12 secretion assay. Mouse BDNF cDNA was subcloned into the pEF4/ Myc-His plasmid vector containing the EF-1a promoter (Invitrogen). Mouse CADPS2 cDNA (GenBank accession number AK038568) was subcloned into the pcDNA3 plasmid vector containing the CMV promoter (Invitrogen) to create pcDNA3-CADPS2(WT). pcDNA3-CADPS2( $\triangle$ exon3) had an internal deletion of 155-265 aa, which corresponded to exon 3 of human CADPS2. Twenty-four hours after transfection with the expression plasmids as described previously (10) using Lipofectamine 2000 Transfection Reagent (Invitrogen), PC12 cells were incubated in fresh assay medium (DMEM containing $0.2 \% \mathrm{BSA}$ ) for 10 minutes. Two different DMEMs were used: standard DMEM (catalog no. 11965; Invitrogen) and high-KCl DMEM (50 mM $\mathrm{KCl} / 65 \mathrm{mM} \mathrm{NaCl}$ based on catalog no. 11965; Invitrogen). Both DMEMs were fully equilibrated in a $5 \% \mathrm{CO}_{2}$ atmosphere at $37^{\circ} \mathrm{C}$ before application. The control and high- $\mathrm{KCl}$ stimulation assay media were collected, and their neurotrophin contents were measured using the BDNF $\mathrm{E}_{\max }$ ImmunoAssay System (Promega) according to the manufacturer's instructions.

Yeast 2-bybrid analysis. The yeast 2-hybrid system (Matchmaker TwoHybrid System 3; Clontech) was used to identify proteins that bind to the exon 3-containing region of mouse CADPS2 (134-331 aa of mouse CADPS2 [GenBank accession number BAD05017]). The aa sequence is almost identical to the $98-295$ aa sequence (Figure 12A) of human CADPS2 (GenBank accession number NP_060424). The partial mouse CADPS2 cDNA (134-331 aa) was cloned in vector pGBKT7 (pGBKT7CADPS2 [134-331 aa]) and used as the bait in yeast 2-hybrid screening. The bait plasmid pGBKT7-CADPS2 (134-331 aa) was transformed into yeast strain AH109 and plated on an SD-Trp plate. An overnight culture (concentrated culture) of positive pGBKT7-CADPS2 (134-331 aa) bait strain in SD/-Trp medium was used to mate with $1 \mathrm{ml}$ of pretransformed adult mouse whole-brain cDNA library according to the manufacturer's instructions. The mating mixture was plated on 50 Quadruple Dropout Medium (QDO; SD/-Ade/-His/-Leu/-Trp/X- $\alpha$-Gal) plates (BD Biosciences) $\left(150 \mathrm{~mm}^{2}, 200 \mu \mathrm{l}\right.$ per plate). Plates were incubated at $30^{\circ} \mathrm{C}$ for up to 14 days. Approximately $18 \times 10^{6}$ colonies were screened. Ten positive colonies were picked and processed for plasmid preparation according to the Clontech yeast plasmid protocol. The resulting yeast plasmids were transformed into E. coli, purified, and sequenced.

Immunoprecipitation and immunoblotting. Mouse CADPS2 cDNA (GenBank accession number AK038568) was subcloned into the pEF-BOS plasmid vector containing the EF-1a promoter (59) with the C-terminal HA primer to create PEF-BOS-CADPS2(WT)-HA. The pEF-BOSCADPS2(Aexon3)-HA had an internal deletion of 155-265 aa, which corresponds to the human CADPS 2 exon 3 coding sequence. The cDNA fragment coding for aas 951-1281 of mouse $\mathrm{p} 150^{\text {Glued }}$ (GenBank accession number NP_031861) was cloned into pEF-BOS with N-terminal FLAG primer to create PEF-BOS-FLAG-p150 Glued (951-1281 aa). COS-7 cells were cultured in DMEM supplemented with $10 \% \mathrm{FBS}$ at $37^{\circ} \mathrm{C}$ and $5 \% \mathrm{CO}_{2}$, and $5 \times 10^{5}$ cells in 6 -well plates were transiently transfected with $1.25 \mathrm{mg}$ pEF-BOS-CADPS2-HA and $3.75 \mathrm{mg}$ pEF-BOS-FLAG-p150 Glued (951-1281 aa) plasmids by using Lipofectamine 2000 Transfection Reagent (Invitrogen). At 24 hours after transfection, COS-7 cells were harvested and lysed in $1.3 \mathrm{ml}$ of Nonidet P40 lysis buffer $(10 \mathrm{mM}$ Tris-HCl, pH 7.5, $150 \mathrm{mM} \mathrm{NaCl}, 1 \mathrm{mM}$ EDTA, and 1\% Nonidet P40) supplemented with Complete protease inhibitor cocktail (Roche). After preabsorption with protein G-sepharose, the supernatants were incubated with $0.5 \mathrm{mg}$ anti-HA antibody or anti-FLAG antibody, and the immunocomplexes were then associated with protein G-sepharose. The resins were washed 5 times with lysis buffer, and the bound proteins were separated on SDS-PAGE gel and transferred to a nitrocellulose membrane for analysis by Western blotting with anti-HA or anti-FLAG antibody.

Cerebellar primary culture. Cerebellar primary cultures were prepared basically as described in a previous study (10). In brief, after rapid decapitation, the P0 cerebella of C57BL/ 6 mice were dissected, digested with $0.1 \%$ Trypsin (Sigma-Aldrich) and $0.05 \%$ DNase I (Roche) in $\mathrm{Ca}^{2+} / \mathrm{Mg}^{2+}$ free HBSS-CaMg(-) (Sigma-Aldrich) for 13 minutes at $37^{\circ} \mathrm{C}$, washed with HBSS-CaMg(-), triturated by repeated passage through a $1-\mathrm{ml}$ plastic micropipette tip in HBSS-CaMg(-) containing $0.05 \%$ DNase I and $12 \mathrm{mM} \mathrm{MgSO}_{4}$, and washed with the following medium: serumfree Eagle's minimal essential medium-based chemical-conditioned medium supplemented with $0.25 \%$ (wt/vol) glucose (Nacalai Tesque), $10 \mu \mathrm{g} / \mathrm{ml}$ insulin (Sigma-Aldrich), $0.1 \mathrm{nM}$ L-thyroxine (Sigma-Aldrich), $0.1 \mathrm{mg} / \mathrm{ml}$ apotransferrin (Sigma-Aldrich), $1 \mathrm{mg} / \mathrm{ml} \mathrm{BSA}$ (Sigma-Aldrich), $2 \mathrm{mM}$ L-glutamine (Nacalai Tesque), $1 \mu \mathrm{g} / \mathrm{ml}$ aprotinin (Sigma-Aldrich), $30 \mathrm{nM}$ sodium selenite (Merck), $100 \mathrm{U} / \mathrm{ml}$ penicillin (Banyu Pharmaceutical Co.), and $135 \mu \mathrm{g} / \mathrm{ml}$ streptomycin (Meiji Seika KK). The dissociated cells were plated at $5 \times 10^{5}$ cells per glass coverslip (12 mm in diameter; Matsunami) coated with poly-L-lysine (Sigma-Aldrich). They were then cultured in the medium under a humidified $5 \% \mathrm{CO}_{2}$ atmosphere at $37^{\circ} \mathrm{C}$. Either CADPS2(WT)-HA or CADPS2( $\Delta$ exon3)-HA tagged with a $\mathrm{C}$-terminal HA epitope was exogenously expressed by the calcium phosphate method at 2 DIV (10). 
Preparation and infection of recombinant adenoviruses. A replication deficient adenovirus Ad-BDNF-GFP was generated by the cosmid-terminal protein complex (COS-TPC) method (60). Briefly, the full-length mouse BDNF cDNA C-terminal tagged with GFP was inserted into the CAG promoter expression unit of pAxCAwt cosmid cassette (Takara Bio Inc.). Recombinant viruses were generated by homologous recombination between EcoT22I-digested Ad5-dlx DNA-terminal protein complex and recombinant cosmid vectors in HEK293 cells as described elsewhere (61). The generated recombinant adenoviruses were propagated in HEK293 cells, then concentrated and purified by double $\mathrm{CsCl}$ step gradient centrifugation. The virus titers were measured on HEK293 cells. The resulting Ad-BDNF-GFP was used for infection of primary cerebellar cultures at an MOI of 30 for 1 hour at $37^{\circ} \mathrm{C}$. As a control, Ad-GFP (26), which expresses GFP, was used.

Secretion assay in cerebellar primary cultures. Cerebellar cultures at 48 hours after infection were incubated for 30 minutes in the chemical-conditioned medium described above. After the incubation, 3 different stimulation assay media were applied for 15 minutes: the standard chemical-conditioned medium described above, high-KCl chemical-conditioned medium $(50 \mathrm{mM} \mathrm{KCl})$, and chemical-conditioned medium with $1 \mathrm{mM}$ TTX and $10 \mathrm{mM}$ NBQX. All media were fully equilibrated in a $5 \% \mathrm{CO}_{2}$ atmosphere at $37^{\circ} \mathrm{C}$ before application. The stimulation assay media were collected, and their BDNF content was measured using the BDNF $\mathrm{E}_{\max }$ ImmunoAssay System (Promega) according to the manufacturer's instructions. BDNF content in cell lysate was also measured using lysis buffer $(20 \mathrm{mM}$ Tris, pH 8.0, $137 \mathrm{mM} \mathrm{NaCl}, 1 \%$ Nonidet P40, and 10\% glycerol) supplemented with Complete protease inhibitor cocktail (Roche).

Neocortical primary culture. Pregnant C57BL/6 mice were anesthetized, and E16 embryos were collected. After rapid decapitation, the neocortices were dissected from the E16 brains and digested with $45 \mathrm{U}$ papain (Worthington), $0.01 \%$ DNase I, $0.02 \%$ DL-cysteine, $0.02 \%$ BSA, and $0.5 \%$ glucose in PBS(-) for 10 minutes at $37^{\circ} \mathrm{C}$. The culture medium was then supplemented with FBS to a final concentration of $20 \%$ and triturated by repeated passage though a $1-\mathrm{ml}$ plastic micropipette tip. The dispersed cells were plated at a density of $5 \times 10^{4} \mathrm{cells} / \mathrm{cm}^{2}$ onto a poly-L-lysine-coated glass coverslip in a neurobasal medium (Invitrogen) containing 2\% B27 supplement (Invitrogen), $500 \mathrm{mM}$ L-glutamine, $0.1 \mathrm{mg} / \mathrm{ml}$ streptomycin, and $100 \mathrm{U} / \mathrm{ml}$ penicillin and cultured under a humidified atmosphere of $5 \% \mathrm{CO}_{2}$ in air at $37^{\circ} \mathrm{C}$.

Secretion assay in neocortical primary cultures. Transfection of neocortical neurons was carried out by the calcium phosphate method using a CellPhect Transfection Kit (Amersham Biosciences) according to the manufacturer's instructions with minor modifications. Full-length CADPS2 [CADPS(WT)] and the exon 3 deletion variant [CADPS2( $\Delta$ exon 3$)]$ were $\mathrm{HA}$ tagged at the $\mathrm{C}$-terminal end and were used for transfection. The reaction mixture, containing $1 \mu \mathrm{g}$ of DNA for each cDNA construct, was added to neocortical primary dissociation cell cultures $\left(5 \times 10^{4}\right.$ cells in $1.9 \mathrm{~cm}^{2}$ culture dish) at 4 DIV in a serum-free neurobasal medium. Cells were then incubated for 30 minutes at $1 \% \mathrm{CO}_{2}$ and $37^{\circ} \mathrm{C}$ followed by rinsing twice with prewarmed neurobasal medium. The transfection efficiency was approximately $30 \%-50 \%$. The BDNF content of culture media collected at 21 DIV was measured using the BDNF $E_{\max }$ ImmunoAssay System (Promega) according to the manufacturer's instructions. The amounts of BDNF, NGF, and NT-3 in the 21 DIV media of untransfected cultures were also measured using the BDNF $\mathrm{E}_{\max }$ ImmunoAssay System (Promega), NGF $E_{\max }$ ImmunoAssay System (Promega), and a highly sensitive 1-site enzyme immunoassay $(10,62)$, respectively.

Statistics. Statistical analysis was performed using Statcel software (OMS). Data were compared using the 2-tailed Student's $t$ test or the Mann-Whitney $U$ test, according to unequal or equal variance. Fisher's exact test was used to compare the prevalence of exon skipping in patients and control subjects. A $P$ value of less than 0.05 was considered statistically significant.

\section{Acknowledgments}

We are grateful to Kazuyuki Yamada, Chieko Nishioka, Tomoko Toyota, Yutaro Komuro, and Bonnie Lee La Madeleine (RIKEN Brain Science Institute) for their technical advice concerning the behavioral tests, excellent technical help in generating the mutant mice, help in human DNA analysis, help in improving our manuscript, and valuable comments on our manuscript, respectively. We thank Sumitomo Pharmaceutical Company for providing human recombinant BDNF. We also thank the Meiji Dairies Corporation for providing the MS12 ES cell line. We are grateful to the families participating in this study. We would also like to thank the Cure Autism Now Foundation for its continued support and operation of the Autism Genetic Resource Exchange resources. This study was supported by grants-in-aid for scientific research from the Japanese Ministry of Education, Culture, Sports, Science, and Technology (grant 17700322 to T. Sadakata), the Japan Society for the Promotion of Science, and RIKEN.

Received for publication May 9, 2006, and accepted in revised form January 16, 2007.

Address correspondence to: Teiichi Furuichi, Laboratory for Molecular Neurogenesis, RIKEN Brain Science Institute, Wako, Saitama 351-0198, Japan. Phone: 81-48-467-6079; Fax: 81-48-4676079; E-mail: tfuruichi@brain.riken.jp.
1. World Health Organization. 1992. The ICD-10 classification of mental and behavioural disorders: clinical descriptions and diagnostic guidelines. World Health Organization. Geneva, Switzerland. http://www.who.int/substance_abuse/terminology/icd_10/en/index.html.

2. American Psychiatric Association. 1994. Diagnostic and statistical manual of mental disorders: DSM-IV. American Psychiatric Publishing Inc. Washington, DC, USA. 886 pP.

3. Muhle, R., Trentacoste, S.V., and Rapin, I. 2004. The genetics of autism. Pediatrics. 113:e472-e486.

4. Folstein, S.E., and Rosen-Sheidley, B. 2001. Genetics of autism: complex aetiology for a heterogeneous disorder. Nat. Rev. Genet. 2:943-955.

5. Jamain, S., et al. 2003. Mutations of the X-linked genes encoding neuroligins NLGN3 and NLGN4 are associated with autism. Nat. Genet. 34:27-29.

6. Kwon, C.H., et al. 2006. Pten regulates neuronal arborization and social interaction in mice. Neuron. 50:377-388.
7. International Molecular Genetic Study of Autism Consortium (IMGSAC). 2001. Further characterization of the autism susceptibility locus AUTS1 on chromosome 7q. Hum. Mol. Genet. 10:973-982.

8. Grishanin, R.N., et al. 2004. CAPS acts at a prefusion step in dense-core vesicle exocytosis as a $\mathrm{PIP}_{2}$ binding protein. Neuron. 43:551-562.

9. Cisternas, F.A., Vincent, J.B., Scherer, S.W., and Ray, P.N. 2003. Cloning and characterization of human CADPS and CADPS2, new members of the $\mathrm{Ca}^{2+}$ dependent activator for secretion protein family. Genomics. 81:279-291.

10. Sadakata, T., et al. 2004. The secretory granuleassociated protein CAPS2 regulates neurotrophin release and cell survival. J. Neurosci. 24:43-52.

11. Sadakata, T., et al. 2006. Differential distributions of the $\mathrm{Ca}^{2+}$-dependent activator protein for secretion family proteins (CAPS2 and CAPS1) in the mouse brain. J. Comp. Neurol. 495:735-753.

12. Bibel, M., and Barde, Y.A. 2000. Neurotrophins: key regulators of cell fate and cell shape in the verte- brate nervous system. Genes Dev. 14:2919-2937.

13. Lu, B. 2003. BDNF and activity-dependent synaptic modulation. Learn. Mem. 10:86-98.

14. Lessmann, V., Gottmann, K., and Malcangio, M. 2003. Neurotrophin secretion: current facts and future prospects. Prog. Neurobiol. 69:341-374.

15. Amir, R.E., et al. 1999. Rett syndrome is caused by mutations in X-linked MECP2, encoding methylCpG-binding protein 2. Nat. Genet. 23:185-188.

16. Chang, Q., Khare, G., Dani, V., Nelson, S., and Jaenisch, R. 2006. The disease progression of Mecp2 mutant mice is affected by the level of BDNF expression. Neuron. 49:341-348.

17. Aman, M.G., and Langworthy, K.S. 2000. Pharmacotherapy for hyperactivity in children with autism and other pervasive developmental disorders. J. Autism Dev. Disord. 30:451-459.

18. Aman, M.G. 2004. Management of hyperactivity and other acting-out problems in patients with autism spectrum disorder. Semin. Pediatr. Neurol. 11:225-228. 
19. Pierce, K., and Courchesne, E. 2001. Evidence for a cerebellar role in reduced exploration and stereotyped behavior in autism. Biol. Psychiatry. 49:655-664.

20. Filipek, P.A., et al. 2000. Practice parameter: screening and diagnosis of autism: report of the Quality Standards Subcommittee of the American Academy of Neurology and the Child Neurology Society. Neurology. 55:468-479.

21. Richdale, A.L., and Prior, M.R. 1995. The sleep/ wake rhythm in children with autism. Eur. Child Adolesc. Psychiatry. 4:175-186.

22. Montgomery, K.C. 1955. The relation between fear induced by novel stimulation and exploratory behavior. J. Comp. Physiol. Psychol. 48:254-260.

23. Casanova, M.F., Buxhoeveden, D., and Gomez, J. 2003. Disruption in the inhibitory architecture of the cell minicolumn: implications for autisim. Neuroscientist. 9:496-507.

24. Jones, K.R., Farinas, I., Backus, C., and Reichardt, L.F. 1994. Targeted disruption of the BDNF gene perturbs brain and sensory neuron development but not motor neuron development. Cell. 76:989-999.

25. Bauman, M., and Kemper, T.L. 1985. Histoanatomic observations of the brain in early infantile autism. Neurology. 35:866-874.

26. Sato, Y., Shiraishi, Y., and Furuichi, T. 2004. Cell specificity and efficiency of the Semliki forest virus vector- and adenovirus vector-mediated gene expression in mouse cerebellum. J. Neurosci. Methods. 137:111-121.

27. Ogawa, H., et al. 2003. A SWI2/SNF2-type ATPase/ helicase protein, $\mathrm{mDomino}$, interacts with myeloid zinc finger protein 2A (MZF-2A) to regulate its transcriptional activity. Genes Cells. 8:325-339.

28. Shibata, N., et al. 2004. Mouse RanBPM is a partner gene to a germline specific RNA helicase, mouse vasa homolog protein. Mol. Reprod. Dev. 67:1-7.

29. Schroer, T.A. 2004. Dynactin. Annu. Rev. Cell Dev. Biol. 20:759-779.

30. Speidel, D., et al. 2003. A family of $\mathrm{Ca}^{2+}$-dependent activator proteins for secretion: comparative analysis of structure, expression, localization, and function. J. Biol. Chem. 278:52802-52809.

31. Waterman-Storer, C.M., et al. 1997. The interaction between cytoplasmic dynein and dynactin is required for fast axonal transport. Proc. Natl. Acad. Sci.U. S. A. 94:12180-12185.

32. Deacon, S.W., et al. 2003. Dynactin is required for bidirectional organelle transport. J. Cell Biol. 160:297-301

33. Burack, M.A., Silverman, M.A., and Banker, G. 2000. The role of selective transport in neuronal protein sorting. Neuron. 26:465-472

34. Detera-Wadleigh, S.D., et al. 1999. A high-density genome scan detects evidence for a bipolar-disorder susceptibility locus on $13 \mathrm{q} 32$ and other poten- tial loci on 1q32 and 18p11.2. Proc. Natl. Acad. Sci. U. S. A. 96:5604-5609.

35. Detera-Wadleigh, S.D., et al. 1997. Initial genome scan of the NIMH genetics initiative bipolar pedigrees: chromosomes 4, 7, 9, 18, 19, 20, and 21q. Am. J. Med. Genet. 74:254-262.

36. Walent, J.H., Porter, B.W., and Martin, T.F. 1992. A novel $145 \mathrm{kd}$ brain cytosolic protein reconstitutes $\mathrm{Ca}^{2+}$-regulated secretion in permeable neuroendocrine cells. Cell. 70:765-775.

37. Berwin, B., Floor, E., and Martin, T.F. 1998. CAPS (mammalian UNC-31) protein localizes to membranes involved in dense-core vesicle exocytosis. Neuron. 21:137-145.

38. Tandon, A., et al. 1998. Differential regulation of exocytosis by calcium and CAPS in semi-intact synaptosomes. Neuron. 21:147-154.

39. Renden, R., et al. 2001. Drosophila CAPS is an essential gene that regulates dense-core vesicle release and synaptic vesicle fusion. Neuron. 31:421-437.

40. Speidel, D., et al. 2005. CAPS1 regulates catecholamine loading of large dense-core vesicles. Neuron. 46:75-88.

41. Brandeis, R., Brandys, Y., and Yehuda, S. 1989. The use of the Morris Water Maze in the study of memory and learning. Int. J. Neurosci. 48:29-69.

42. Dulawa, S.C., Grandy, D.K., Low, M.J., Paulus, M.P., and Geyer, M.A. 1999. Dopamine D4 receptor-knock-out mice exhibit reduced exploration of novel stimuli. J. Neurosci. 19:9550-9556.

43. Levin, E.D., and Bowman, R.E. 1986. Effects of the dopamine D-2 receptor agonist, LY 171555, on radial arm maze performance in rats. Pharmacol. Biochem. Behav. 25:1117-1119.

44. Breese, G.R., and Mueller, R.A. 1985. SCH-23390 antagonism of a D-2 dopamine agonist depends upon catecholaminergic neurons. Eur. J. Pharmacol. 113:109-114.

45. Binda, A.V., Kabbani, N., and Levenson, R. 2005. Regulation of dense core vesicle release from PC12 cells by interaction between the D2 dopamine receptor and calcium-dependent activator protein for secretion (CAPS). Biochem. Pharmacol. 69:1451-1461.

46. Miyazaki, K., et al. 2004. Serum neurotrophin concentrations in autism and mental retardation: a pilot study. Brain Dev. 26:292-295

47. Nelson, K.B., et al. 2001. Neuropeptides and neurotrophins in neonatal blood of children with autism or mental retardation. Ann. Neurol. 49:597-606.

48. Connolly, A.M., et al. 2006. Brain-derived neurotrophic factor and autoantibodies to neural antigens in sera of children with autistic spectrum disorders, Landau-Kleffner syndrome, and epilepsy. Biol. Psychiatry. 59:354-363.
49. Nelson, P.G., et al. 2006. Selected neurotrophins, neuropeptides, and cytokines: developmental trajectory and concentrations in neonatal blood of children with autism or Down syndrome. Int. J. Dev. Neurosci. 24:73-80.

50. Hashimoto, K., et al. 2006. Reduced serum levels of brain-derived neurotrophic factor in adult male patients with autism. Prog. Neuropsychopharmacol. Biol. Psychiatry. 30:1529-1531.

51. Courchesne, E., and Pierce, K. 2005. Why the frontal cortex in autism might be talking only to itself: local over-connectivity but long-distance disconnection. Curr. Opin. Neurobiol. 15:225-230.

52. Chen, W.G., et al. 2003. Derepression of BDNF transcription involves calcium-dependent phosphorylation of MeCP2. Science. 302:885-889.

53. Martinowich, K., et al. 2003. DNA methylationrelated chromatin remodeling in activity-dependent BDNF gene regulation. Science. 302:890-893.

54. Lord, C., Rutter, M., and Le Couteur, A. 1994. Autism Diagnostic Interview-Revised: a revised version of a diagnostic interview for caregivers of individuals with possible pervasive developmental disorders. J. Autism Dev. Disord. 24:659-685.

55. Kawase, E., et al. 1994. Strain difference in establishment of mouse embryonic stem (ES) cell lines. Int. J. Dev. Biol. 38:385-390.

56. Han, B.H., and Holtzman, D.M. 2000. BDNF protects the neonatal brain from hypoxic-ischemic injury in vivo via the ERK pathway. J. Neurosci. 20:5775-5781.

57. Nawa, H., Pelleymounter, M.A., and Carnahan, J. 1994. Intraventricular administration of BDNF increases neuropeptide expression in newborn rat brain. J. Neurosci. 14:3751-3765.

58. Fukuda, D., Sata, M., Tanaka, K., and Nagai, R. 2005. Potent inhibitory effect of sirolimus on circulating vascular progenitor cells. Circulation. 111:926-931.

59. Mizushima, S., and Nagata, S. 1990. pEF-BOS, a powerful mammalian expression vector. Nucleic Acids Res. 18:5322.

60. Miyake, S., et al. 1996. Efficient generation of recombinant adenoviruses using adenovirus DNAterminal protein complex and a cosmid bearing the full-length virus genome. Proc. Natl. Acad. Sci. U.S. A. 93:1320-1324.

61. Kanegae, Y., Makimura, M., and Saito, I. 1994. A simple and efficient method for purification of infectious recombinant adenovirus. Jpn. J. Med. Sci. Biol. 47:157-166.

62. Katoh-Semba, R., Takeuchi, I.K., Semba, R., and Kato, K. 2000. Neurotrophin-3 controls proliferation of granular precursors as well as survival of mature granule neurons in the developing rat cerebellum. J. Neurochem. 74:1923-1930. 